\title{
Danshensu for Myocardial Ischemic Injury: Preclinical Evidence and Novel Methodology of Quality Assessment Tool
}

\section{OPEN ACCESS}

Edited by: Thomas Efferth,

Johannes Gutenberg University

Mainz, Germany

Reviewed by:

Lei Chen

Fujian Agriculture and Forestry

University, China

Pui Kei Wu

Medical College of Wisconsin,

United States

*Correspondence:

Guo-qing Zheng

gq_zheng@sohu.com

Yan Wang

wywzchina@sina.com

Specialty section:

This article was submitted to

Ethnopharmacology,

a section of the journal

Frontiers in Pharmacology

Received: 04 August 2018 Accepted: 22 November 2018 Published: 11 December 2018

Citation:

Bao X, Zheng Q, Tong Q, Zhu P, Zhuang $Z$, Zheng $G$ and Wang $Y$ (2018) Danshensu for Myocardial Ischemic Injury: Preclinical Evidence and Novel Methodology of Quality

Assessment Tool.

Front. Pharmacol. 9:1445. doi: 10.3389/fphar.2018.01445

\section{Xiao-yi Bao ${ }^{1}$, Qun Zheng ${ }^{1}$, Qiang Tong ${ }^{1}$, Peng-chong Zhu ${ }^{1}$, Zhuang Zhuang ${ }^{1}$, Guo-qing Zheng ${ }^{2 *}$ and Yan Wang ${ }^{1 *}$}

\begin{abstract}
${ }^{1}$ Department of Cardiology, The Second Affiliated Hospital and Yuying Children's Hospital of Wenzhou Medical University, Wenzhou, China, ${ }^{2}$ Department of Neurology, The Second Affiliated Hospital and Yuying Children's Hospital of Wenzhou Medical University, Wenzhou, China
\end{abstract}

Background: Danshensu (DSS) possesses unique bioactivity on the cardiovascular system. However, there is a lack of systematical summary of DSS for acute myocardial ischemia injury and no quality assessment tool for the systematical review of cell experiments. Here, we aimed to assess the preclinical evidences and possible mechanisms of DSS for myocardial ischemia injury, and to develop a quality assessment tool for the systematical review of cell experiments.

Methods: Thirty-two studies with 473 animals and 134 cells were identified by searching seven databases. All data analysis was performed using RevMan 5.3. CAMARADES 10-item checklist was used to assess the methodological quality of animal experiments. A new 10-item checklist was first developed to assess the methodological quality of cell studies.

Results: The score of study quality ranged from 3 to 7 points in animal studies, while the cell studies scored 3-6 points. Meta-analysis showed that DSS had significant effects on reducing myocardial infarct (MI) size in vivo, and increasing cell viability and reducing apoptosis rate in vitro compared with controls $(P<0.01)$. The possible mechanisms of DSS for $\mathrm{Ml}$ are improving circulation, antioxidant, anti-apoptosis, anti-inflammatory, promoting angiogenesis, anti-excessive autophagy, anti-calcium overload, and improving energy metabolism.

Conclusions: DSS could exert cardioprotective effect on myocardial ischemia injury, and thus is a probable candidate for further clinical trials andtreatment of AMI. In addition, the newly devloped 10-item checklist for assessing methodological quality of cell study that recommened to use the sysmatic review of cell studies.

Keywords: danshensu, infarct size, ischemia, reperfusion, meta-analysis, methodology 


\section{INTRODUCTION}

Acute myocardial infarction (AMI) is a major cause of death and disability worldwide (Mozaffarian et al., 2016). During AMI, the damage inflicted on the myocardium results in two processes: ischemia and the following reperfusion (I/R) (Ibanez et al., 2015). Thus, timely myocardial reperfusion using either thrombolytic therapy or primary percutaneous coronary intervention (PPCI) is the most effective therapy for limiting myocardial infarct (MI) size, preserving left-ventricular systolic function and reducing the onset of heart failure (Frohlich et al., 2013). However, the mortality and morbidity of patients remain significant with $9 \%$ death and $10 \%$ heart failure at 1 year after AMI. The fact that a therapeutic intervention administered solely at the time of myocardial I/R injury can reduce MI size by up to half only suggests that myocardial I/R injury may account for up to $50 \%$ of the final MI size (Bulluck et al., 2016). Although the process of myocardial reperfusion has been optimized by advances in stent technology, new antiplatelet drugs and novel antithrombotic agents, the studies on drug for necrosis and inflammation of cardiac myocytes during AMI made little evident progress. And there is still no effective therapy for preventing myocardial I/R injury in PPCI patients (Hausenloy and Yellon, 2013). Thus, it is urgent to seek new cardioprotective strategies to improve myocardial salvage and cardiac function when AMI happens.

Phenolic and flavonoid compounds with specific structures exhibit strong activity. For example, agrimonolide, curcumin, emodin, resveratrol, baicalin and meletin exerted antiinflammatory, antioxidant, anti-apoptosis effects and regulated metabolism through multiple intracellular signaling pathways (Chen et al., 2016, 2018a; Teng et al., 2016a,b). Previous studies showed that the application of these compounds is beneficial to various diseases (Chen et al., 2018b; Teng and Chen, 2018). Danshen root, Radix Salviae Miltiorrhizae, the dried root of Salvia miltiorrhiza, has been widely used to treat various vascluar diseases including coronary heart disease worldwide (Zhang et al., 2018). Previous studies showed that one of the main water-soluble phenolic components from Danshen, Danshensu (DSS) [(3-(3,4-dihydroxyphenyl)-lactic acid)], exerted potential cardiovascular protective effects both in vitro and in vivo (Dong et al., 2009; Cui et al., 2014; Yu J. et al., 2015; Li et al., 2016; Yin et al., 2017; Zhang X. et al., 2017). The specific chemical structures of Danshensu and its derivatives are listed in Table 1. Futhermore, preclinical systematic review can evaluate the efficacy and mechanism of drugs more systematically, establish a test field for further animal experiments, provide reliable information for drug research, and lay a foundation for future clinical research (Sena et al., 2014). what's more, there are no quality assessment tools for a systematical review of cell experiments. Thus, we aimed to provide the preclinical evidence by assessing the efficacy and mechanisms of DSS for expermental AMI, and develop a quality assessment tool for a systematical review of cell experiments.

\section{METHODS}

The prensent study was conducted according to the Preferred Reporting Items for Systematic reviews and Meta-Analyses (PRISMA) guidelines (Liberati et al., 2009).

\section{Search Strategies}

The following databases were searched from the inception to February, 2018. They are PubMed, CENTRAL, Web of Science, Chinese National Knowledge Infrastructure, Wanfang Data Information Site, VIP information database and Chinese Biomedical Literature Database. The keywords used are as follows: (1) "Danshensu OR Salvianic acid A OR tanshinol OR 3,4-dihydroxyphenyl" AND "myocardial infarction OR myocardial ischemia OR myocardial I/R" limited on animals; (2) "Danshensu OR Salvianic acid A OR tanshinol OR 3,4dihydroxyphenyl" AND "hypoxia OR injury OR damage OR impair" limited on cells. The literature search in this article was last updated in February, 2018.

\section{Eligibility Criteria}

Inclusion criteria were prespecified as follows: (1) experimental animal models of myocardial ischemia or myocardial cell injury models; (2) analyzed treatments received the DSS [to enhance stability and activities of danshensu, Danshensu derivatives (Dong et al., 2009; Cui et al., 2014; Zhang X. et al., 2017) have been widely developed and thus was also included]; (3) comparator interventions received vehicle or non-functional liquid with equal volume or conventional medicine or no treatment; (4) the primary outcome measures were MI size in animal model, and the viability and/or apoptosis rate in cell model. The second outcome measures were biochemical markers of MI, left ventricular ejection fraction (LVEF), left ventricular shortening fraction (LVFS), and/or level of STsegment depression, and mechanism indices. Exclusion criteria were prespecified as follows: (1) experimental models unrelated to myocardial ischemic injury; (2) the use of DSS in combination with other drugs in treatment group; (3) no control group or comparing DSS with other herbs in control group; (4) duplicate publication; (5) case report, clinical trial and review article.

\section{Data Extraction}

The detailed information of included studies were collected by two indenpendent authors. For animal studies, standard extraxted form on the basis of our previous publication was adopted (Zhang K. J. et al., 2017). For cell studies, the information extracted from included studies was as follows: (1) the first author's name and year of publication; (2) the features of cells, such as name, source (organism, age, tissue) and primary/subcultured; (3) model of cell injury; (4) the information of treatment/control group, including therapeutic drug, dosage, method of administration, duration of treatment; (5) mean value and standard deviation of outcomes.

If the outcomes were displayed through multiple time points, only the peak time point was included. Only the data of highest dose was selected, if the experimental group included gradient doses of drug therapy. Owing to some data were only displayed by graphics, the correspondence authors were be contacted for further information. If there were no responding, the data in the graph were measured by the digital ruler software. 
TABLE 1 | Specific chemical structures of Danshensu and its derivatives.

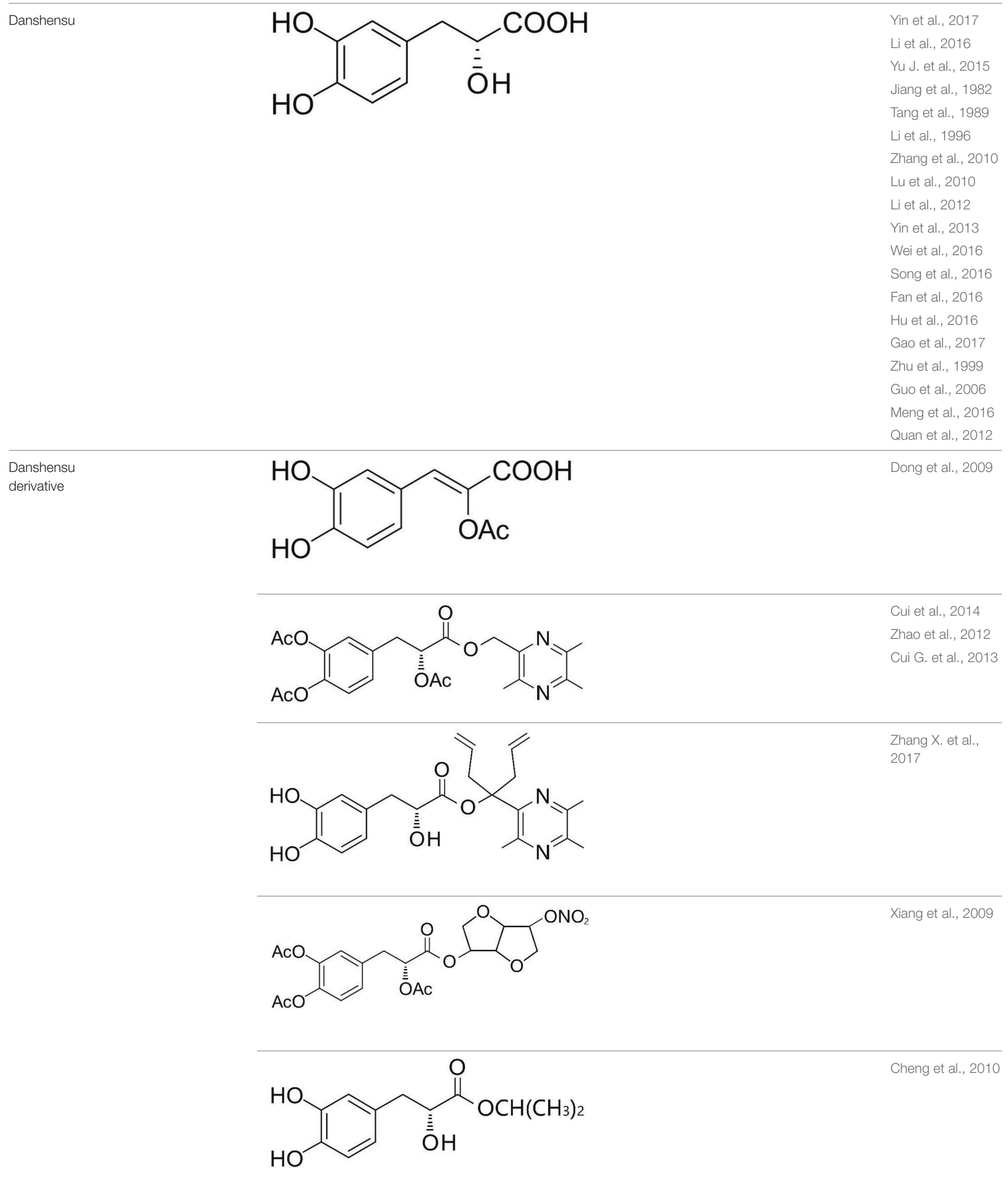


<smiles>C=CCC(CC=C)(OC(=O)[C@H](Cc1ccc(OC(=O)CN)c(OC(=O)CN)c1)OC(=O)c1ccc(-c2csc(=S)s2)cc1)c1nc(C)c(C)nc1C</smiles><smiles>C=CCC(CC=C)(OC(=O)[C@H](Cc1ccc(OC(C)=O)c(OC(C)=O)c1)OC(=O)CN)c1nc(C)c(C)nc1C</smiles><smiles>C=CCC(CC=C)(OC(=O)[C@@H](Cc1ccc(O)c(O)c1)OC(C)=O)c1nc(C)c(C)nc1C</smiles>

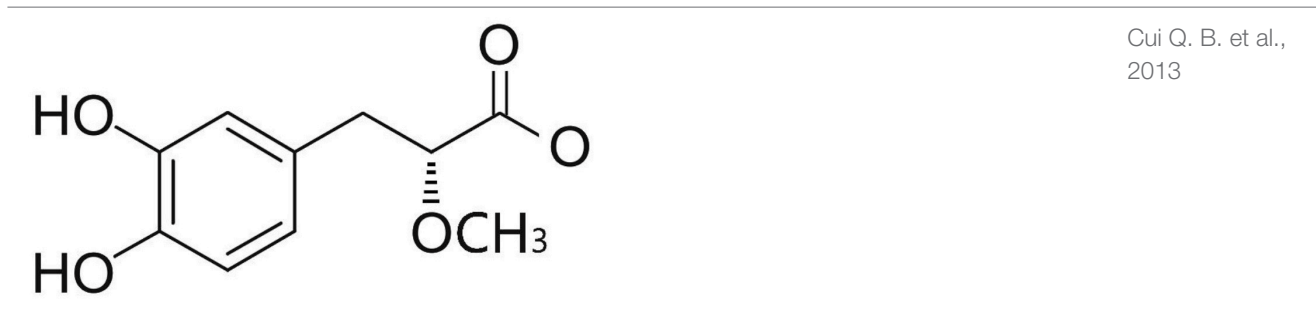<smiles>C=COC(=O)[C@H](Cc1ccc(OC(C)=O)c(OC(C)=O)c1)OC(C)=O</smiles>

\section{Risk of Bias in Individual Studies}

The risk of bias (ROB) in animal studies was refered to the ten-item scale using our previously publications (Zhang K. J. et al., 2017). Owing to absence of the ROB assessment scale in cell experiments, we developed a novel 10-item checklist as follows: (A) peer reviewed publication; (B) use appropriate cells to study; (C) cell lines with reliable source or validated by appropriate methods; (D) assess toxicity of treatment on cells; (E) culture environment (culture media/sera, $\mathrm{pH} / \mathrm{CO}_{2}$ and temperature); (F) random allocation to treatment or control; 
(G) blinded induction of model; $(\mathrm{H})$ blinded assessment of outcome; (I) calculation of the sample size necessary to achieve sufficient power; and (J) statement of potential conflict of interests. Each item was awarded one point. All studies were evaluated by two independent authors. Possible divergences in the evaluation process were properly addressed through contacting with correspondence authors.

\section{Statistical Analysis}

All data analysis was implemented by RevMan 5.3. We calculated the standard mean difference (SMD) with 95\% Confidence Intervals (CIs). Heterogeneity was assessed using the Cochrane Q-statistic test and the $I^{2}$-statistic test. When $I^{2}>50 \%$, indicating substantial heterogeneity and a random-effects model was adopted. Conversely, a fixed-effects model was used. Sources of heterogeneity were searched as far as possible and subgroup analysis was carried out when necessary. Funnel plots were used to intuitively reflect publication bias when more than ten studies were identified. Sensitivity analysis was carried out to prove the robustness of our main results. $P<0.05$ was considered statistically significant.

\section{RESULTS}

\section{Study Selection}

For animal studies, 637 potentially relevant studies were identified through database searching, of which 550 were duplicated. After preliminary screening of the titles and abstracts, we excluded 41 studies because of case report, clinical observation, and review article. Then secondary screening was conducted by reading the full text of the remaining 46 studies, and 19 studies were excluded because of at least one of the following reasons: (1) failed to obtain full text; (2) not DSS intervention; (3) inappropriate animal model; (4) combined use of other drugs in treatment group; (5) compared with herbs or active herbal compounds; (6) no control group; (7) master dissertation or doctoral dissertation; (8) no available data. Ultimately, 27 eligible studies (Jiang et al., 1982; Tang et al., 1989, 2018; Li et al., 1996, 2012, 2016; Dong et al., 2009; Xiang et al., 2009; Cheng et al., 2010; Lu et al., 2010; Zhang et al., 2010; Quan et al., 2012; Zhao et al., 2012; Cui G. et al., 2013; Yin et al., 2013, 2017; Chen et al., 2014; Cui et al., 2014; Yu J. et al., 2015; Fan et al., 2016; Hu et al., 2016; Song et al., 2016; Wang et al., 2016, 2017; Wei et al., 2016; Gao et al., 2017; Zhang X. et al., 2017) were selected (Figure 1A).

For cell studies, 257 potentially relevant studies were identified through database searching, of which 39 were duplicated. After preliminary screening of the titles and abstracts, we excluded 14 studies because of case report, clinical observation, and review article. Then secondary screening was conducted by reading the full text of the remaining 25 studies, nine studies were excluded because of: (1) failed to obtain full text; (2) not DSS intervention; (3) inappropriate cell model; (4) combined use of other drugs in treatment group; (5) compared with herbs or active herbal compounds; (6) no control group; (7) master dissertation or doctoral dissertation; (8) no available data. Ultimately, 16 eligible studies (Zhu et al., 1999; Guo et al., 2006; Dong et al., 2009; Zhao et al., 2012; Cui G. et al., 2013; Cui Q. B. et al., 2013; Xu et al., 2013; Yin et al., 2013; Fan et al., 2016; Hu et al., 2016; Meng et al., 2016; Wang et al., 2016, 2017; Gao et al., 2017; Zhang X. et al., 2017; Tang et al., 2018) were selected (Figure 1B).

\section{Characteristics of Included Studies Animal Experiments}

Twenty-seven studies were involved in animal experiments. Eleven studies were published in chinese (Jiang et al., 1982; Tang et al., 1989; Li et al., 1996; Xiang et al., 2009; Cheng et al., 2010; Lu et al., 2010; Zhang et al., 2010; Quan et al., 2012; Zhao et al., 2012; Chen et al., 2014; Wei et al., 2016), and the rest was published in English (Dong et al., 2009; Li et al., 2012, 2016; Cui G. et al., 2013; Yin et al., 2013, 2017; Yu J. et al., 2015; Fan et al., 2016; Hu et al., 2016; Song et al., 2016; Wang et al., 2016, 2017; Gao et al., 2017; Zhang X. et al., 2017; Tang et al., 2018) between 1982 and 2018. Sprague-Dawley (SD) male rats were used in 21 studies (Dong et al., 2009; Xiang et al., 2009; Lu et al., 2010; Zhang et al., 2010; Li et al., 2012, 2016; Quan et al., 2012; Zhao et al., 2012; Cui G. et al., 2013; Yin et al., 2013, 2017; Chen et al., 2014; Cui et al., 2014; Yu J. et al., 2015; Fan et al., 2016; Hu et al., 2016; Song et al., 2016; Wei et al., 2016; Gao et al., 2017; Wang et al., 2017; Zhang X. et al., 2017), two that used male Wistar rats (Tang et al., 1989; Li et al., 1996), one that used both female and male Wistar rats (Cheng et al., 2010), two that used Zebrafish embryos (Wang et al., 2016; Tang et al., 2018) and one that used male canines with unknow species (Jiang et al., 1982). The weight of the rats were $180-320 \mathrm{~g}$, and the canines were $12-26 \mathrm{~kg}$. To induce anesthesia, 16 studies used nembutal (Jiang et al., 1982; Tang et al., 1989; Li et al., 1996, 2012, 2016; Xiang et al., 2009; Cheng et al., 2010; Quan et al., 2012; Yin et al., 2013, 2017; Chen et al., 2014; Yu J. et al., 2015; Fan et al., 2016; Hu et al., 2016; Wang et al., 2017; Zhang X. et al., 2017) seven studies used chloral hydrate (Dong et al., 2009; Zhang et al., 2010; Zhao et al., 2012; Cui G. et al., 2013; Cui et al., 2014; Wei et al., 2016; Gao et al., 2017); and two studies used ethyl carbamate (Lu et al., 2010; Song et al., 2016). Fifteen MI models were produced by ligation of the left anterior descending coronary artery (LAD) (Jiang et al., 1982; Dong et al., 2009; Xiang et al., 2009; Lu et al., 2010; Quan et al., 2012; Zhao et al., 2012; Cui G. et al., 2013; Yin et al., 2013, 2017; Chen et al., 2014; Cui et al., 2014; Hu et al., 2016; Wei et al., 2016; Wang et al., 2017; Zhang X. et al., 2017) seven models produced by the langendorff method (Tang et al., 1989; Li et al., 1996; Cheng et al., 2010; Zhang et al., 2010; Yu J. et al., 2015; Fan et al., 2016; Gao et al., 2017); three models produced by subcutaneous injection of Isoproterenol (ISO) (Li et al., 2012, 2016; Song et al., 2016); and the remain two by exposure to Doxorubicin (Dox) (Wang et al., 2016; Tang et al., 2018). For outcome measures, 16 studies reported the MI size (Jiang et al., 1982; Dong et al., 2009; Xiang et al., 2009; Lu et al., 2010; Li et al., 2012; Quan et al., 2012; Cui G. et al., 2013; Yin et al., 2013, 2017; Chen et al., 2014; Cui et al., 2014; Yu J. et al., 2015; Hu et al., 2016; Wei et al., 2016; Wang et al., 2017; Zhang X. et al., 2017) level of ST-segment change (Li et al., 2012), LVEF or LVFS (Yin et al., 2017) was reported in one study, respectively. Lactate dehydrogenase $(\mathrm{LDH})$ was reported in eight studies (Dong et al., 2009; Xiang et al., 2009; Lu et al., 2010; Li et al., 2012; Cui et al., 2014; Yu J. et al., 2015; Fan et al., 


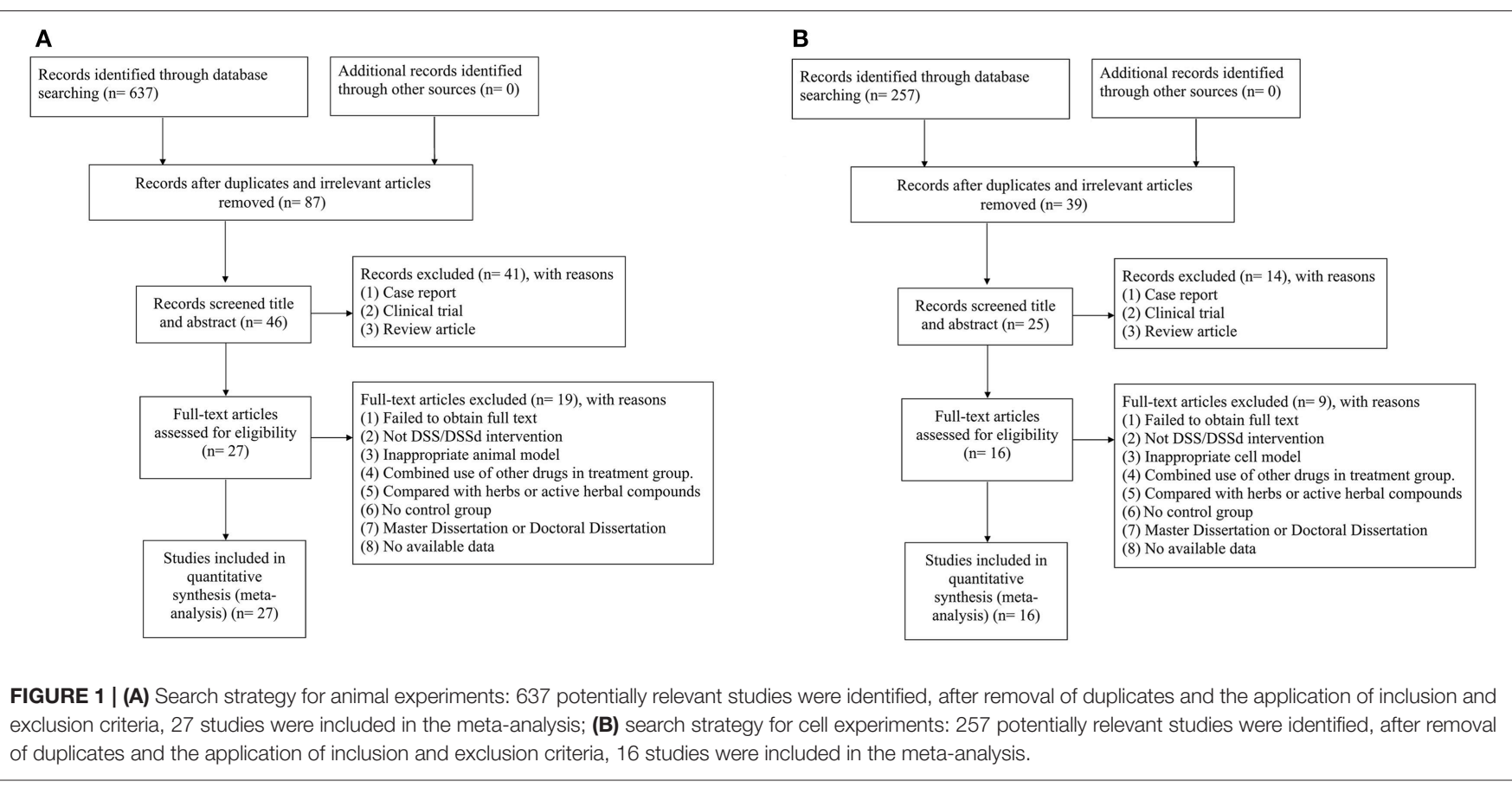

2016; Song et al., 2016), creatine kinase (CK) in seven studies (Dong et al., 2009; Xiang et al., 2009; Lu et al., 2010; Cui et al., 2014; Yu J. et al., 2015; Fan et al., 2016; Song et al., 2016), creatine kinase-MB (CK-MB) in five studies (Li et al., 2012, 2016; Quan et al., 2012; Yin et al., 2013; Hu et al., 2016), cardiac troponin-T (cTnT) in one study (Li et al., 2016). The overall characteristics of included publications are shown in Table 2.

\section{Cell Experiments}

Sixteen studies were involved in cell experiments. Five studies were published in chinese (Zhu et al., 1999; Guo et al., 2006; Zhao et al., 2012; Cui Q. B. et al., 2013; Xu et al., 2013), and the rest was published in English (Dong et al., 2009; Cui G. et al., 2013; Yin et al., 2013; Fan et al., 2016; Hu et al., 2016; Meng et al., 2016; Wang et al., 2016, 2017; Gao et al., 2017; Zhang X. et al., 2017; Tang et al., 2018) between 1999 and 2017. H9c2 cells (Zhao et al., 2012; Cui G. et al., 2013; Cui Q. B. et al., 2013; Xu et al., 2013; Yin et al., 2013; Hu et al., 2016; Meng et al., 2016; Wang et al., 2016, 2017; Gao et al., 2017; Zhang X. et al., 2017; Tang et al., 2018), neonatal SD rat cardiomyocytes (Dong et al., 2009; Fan et al., 2016), adult wistar rat cardiomyocytes (Zhu et al., 1999), and neonatal wistar rat cardiomyocytes (Guo et al., 2006) were used in 12, 2, 1, and 1 studies/study, respectively. Four kinds of cardiomyocytes injured models were induced by hypoxia/reoxygenation (H/R) (Zhu et al., 1999; Fan et al., 2016; Hu et al., 2016; Meng et al., 2016), 1 by hypoxia (Dong et al., 2009), 1 by ischemia-H/R (Yin et al., 2013), 6 by tert-Butyl hydroperoxide (t-BHP) (Zhao et al., 2012; Cui G. et al., 2013; Cui Q. B. et al., 2013; Xu et al., 2013; Wang et al., 2017; Zhang X. et al., 2017), 2 by Dox (Wang et al., 2016; Tang et al., 2018), 1 by angiotensin II (AngII) (Guo et al., 2006) and 1 by oxygen glucose deprivation (Gao et al., 2017). For outcome measures, 12 studies used the cell viability (Dong et al., 2009; Cui G. et al., 2013; Cui Q. B. et al., 2013; Xu et al., 2013; Yin et al., 2013; Fan et al., 2016; Hu et al., 2016; Wang et al., 2016, 2017; Gao et al., 2017; Zhang X. et al., 2017; Tang et al., 2018), 11 that used apoptosis rate (Guo et al., 2006; Zhao et al., 2012; Cui G. et al., 2013; Yin et al., 2013; Hu et al., 2016; Meng et al., 2016; Wang et al., 2016, 2017; Gao et al., 2017; Zhang X. et al., 2017; Tang et al., 2018), six that used LDH (Dong et al., 2009; Cui G. et al., 2013; Yin et al., 2013; Hu et al., 2016; Wang et al., 2016; Tang et al., 2018). The overall characteristics of included studies are shown in Table 3.

\section{Study Quality \\ Animal Studies}

The quality score of included studies ranged from 3 to 7 . All studies obtained two points from peer-reviewed publication, and anesthetic without significant cardioprotective (Jiang et al., 1982; Tang et al., 1989, 2018; Li et al., 1996, 2012, 2016; Dong et al., 2009; Xiang et al., 2009; Cheng et al., 2010; Lu et al., 2010; Zhang et al., 2010; Quan et al., 2012; Zhao et al., 2012; Cui G. et al., 2013; Yin et al., 2013, 2017; Chen et al., 2014; Cui et al., 2014; Yu J. et al., 2015; Fan et al., 2016; Hu et al., 2016; Song et al., 2016; Wang et al., 2016, 2017; Wei et al., 2016; Gao et al., 2017; Zhang X. et al., 2017). Twenty-three studies reported random allocation (Jiang et al., 1982; Dong et al., 2009; Xiang et al., 2009; Cheng et al., 2010; Lu et al., 2010; Zhang et al., 2010; Li et al., 2012, 2016; Quan et al., 2012; Zhao et al., 2012; Cui G. et al., 2013; Yin et al., 2013, 2017; Chen et al., 2014; Cui et al., 2014; Yu J. et al., 2015; Fan et al., 2016; Hu et al., 2016; Song et al., 2016; Wei et al., 2016; Gao et al., 2017; Wang et al., 2017; Zhang X. et al., 2017). None of studies mentioned blinded assessment of outcome, described a sample size calculation, or used appropriate animal model. One study used a blind method in modeling (Yin et al., 2017). Twenty-four 


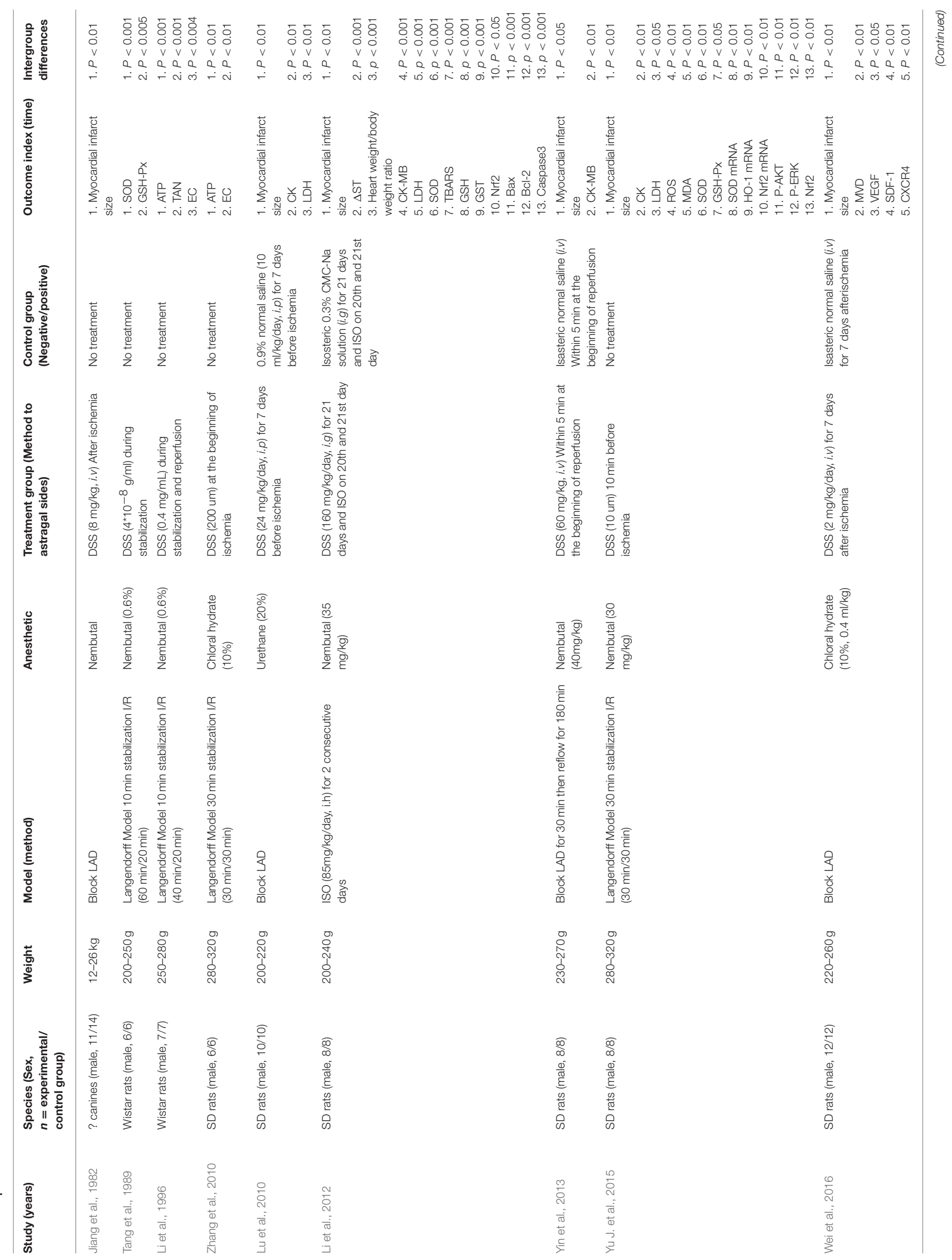




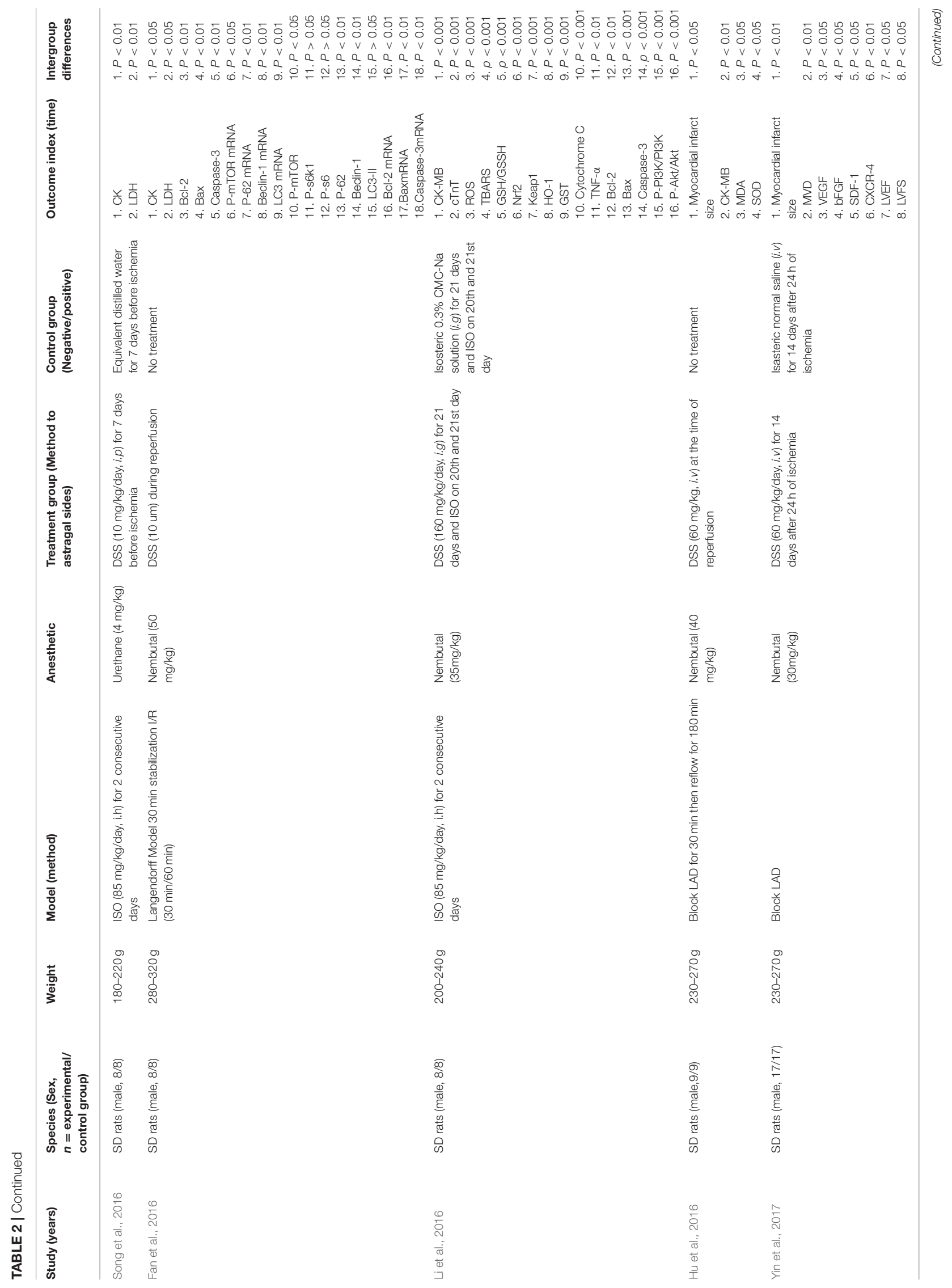




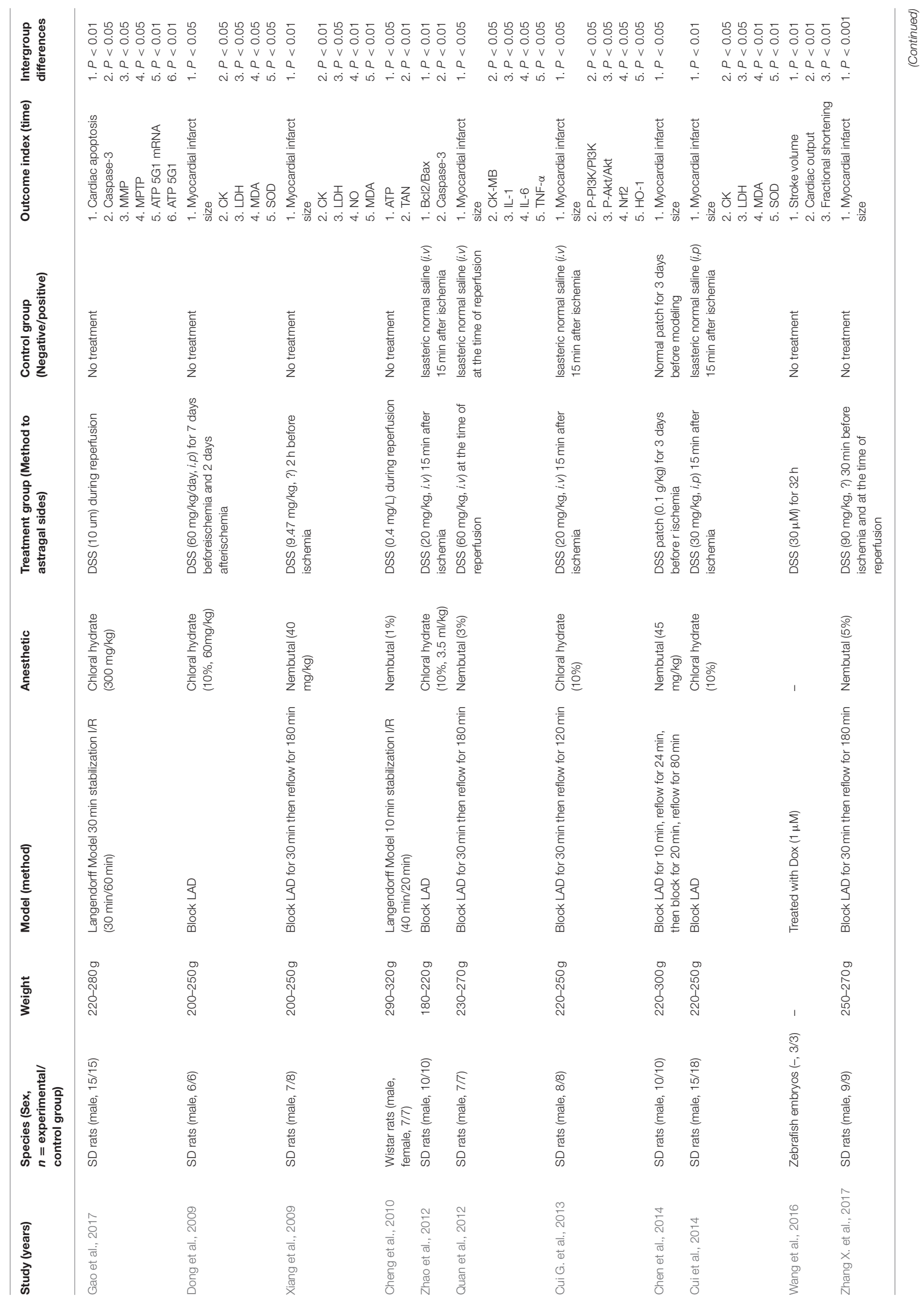




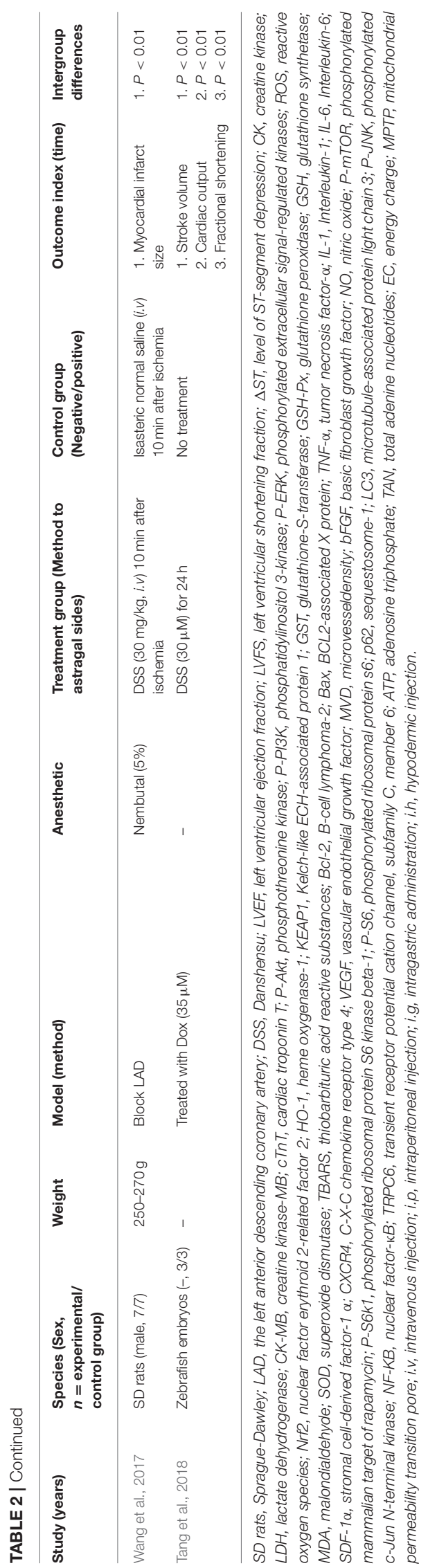

studies reported the control of temperature (Tang et al., 1989, 2018; Li et al., 1996, 2012, 2016; Dong et al., 2009; Xiang et al., 2009; Cheng et al., 2010; Zhang et al., 2010; Quan et al., 2012; Zhao et al., 2012; Cui G. et al., 2013; Yin et al., 2013, 2017; Chen et al., 2014; Cui et al., 2014; Yu J. et al., 2015; Fan et al., 2016; Hu et al., 2016; Song et al., 2016; Wang et al., 2016, 2017; Wei et al., 2016; Gao et al., 2017), 13 studies complianced with animal welfare regulations (Li et al., 2012, 2016; Cui G. et al., 2013; Yin et al., 2013, 2017; Yu J. et al., 2015; Fan et al., 2016; Hu et al., 2016; Song et al., 2016; Wang et al., 2016, 2017; Gao et al., 2017; Tang et al., 2018) and 17 studies declared the potential conflict of interests (Dong et al., 2009; Zhang et al., 2010; Li et al., 2012, 2016; Cui G. et al., 2013; Yin et al., 2013, 2017; Cui et al., 2014; Yu J. et al., 2015; Fan et al., 2016; Hu et al., 2016; Song et al., 2016; Wang et al., 2016, 2017; Gao et al., 2017; Zhang X. et al., 2017; Tang et al., 2018). The methodological quality of each study was summarized in Table 4.

\section{Cell Studies}

The quality score of included studies ranged from 3 to 6 . All the studies obtained two points from peer-reviewed publication, and culture environment (Zhu et al., 1999; Guo et al., 2006; Dong et al., 2009; Zhao et al., 2012; Cui G. et al., 2013; Cui Q. B. et al., 2013; Xu et al., 2013; Yin et al., 2013; Fan et al., 2016; Hu et al., 2016; Meng et al., 2016; Wang et al., 2016, 2017; Gao et al., 2017; Zhang X. et al., 2017; Tang et al., 2018). No study described a sample size calculation. One study used use primary adult cardiomyocytes (Zhu et al., 1999), three studies exameid the toxicity of treatment on cells (Cui G. et al., 2013; Wang et al., 2016; Tang et al., 2018) and four stuides mentioned random allocation to treatment or control (Zhao et al., 2012; Xu et al., 2013; Yin et al., 2013; Hu et al., 2016). Seven studies reported cell lines with reliable source or validated by appropriate methods (Guo et al., 2006; Dong et al., 2009; Cui G. et al., 2013; Yin et al., 2013; Fan et al., 2016; Hu et al., 2016; Meng et al., 2016). Twelve studies declared the potential conflict of interests (Dong et al., 2009; Cui G. et al., 2013; Cui Q. B. et al., 2013; Yin et al., 2013; Fan et al., 2016; Hu et al., 2016; Meng et al., 2016; Wang et al., 2016, 2017; Gao et al., 2017; Zhang X. et al., 2017; Tang et al., 2018). The methodological quality of each study was summarized in Table 5.

\section{Effectiveness MI Size}

Meta-analysis of 15 studies (Dong et al., 2009; Xiang et al., 2009; Lu et al., 2010; Li et al., 2012; Quan et al., 2012; Cui G. et al., 2013; Yin et al., 2013, 2017; Chen et al., 2014; Cui et al., 2014; Yu J. et al., 2015; Hu et al., 2016; Wei et al., 2016; Wang et al., 2017; Zhang X. et al., 2017) showed DSS has significant effects on reducing MI size compared with control ( $n=231$, SMD: $-2.27,95 \% \mathrm{CI}:-2.65$ to $-1.89, P<0.00001$; heterogeneity $\chi^{2}=52.89, P<0.00001$, $\left.I^{2}=74 \%\right)$. We conducted subgroup analysis according to the different animal models because of high heterogeneity. Metaanalysis of seven studies (Xiang et al., 2009; Quan et al., 2012; Cui G. et al., 2013; Yin et al., 2013; Yu J. et al., 2015; Hu et al., 2016; Zhang X. et al., 2017) showed more significant effect than that of controls according to rat models of I/R $(n=96$, SMD: -2.94 , $95 \%$ CI: -3.60 to $-2.27, P<0.00001$; heterogeneity $\chi^{2}=11.62$, 


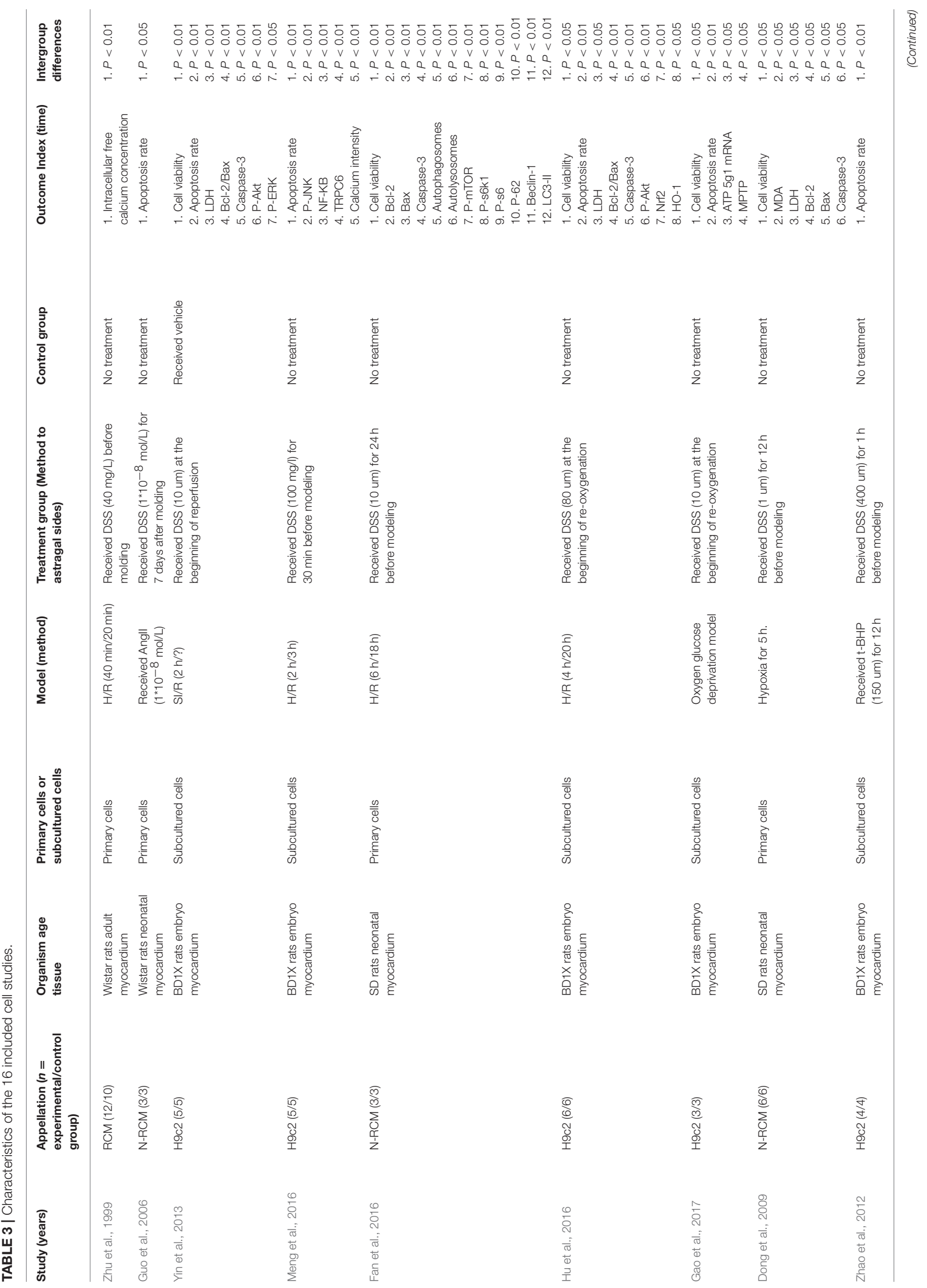




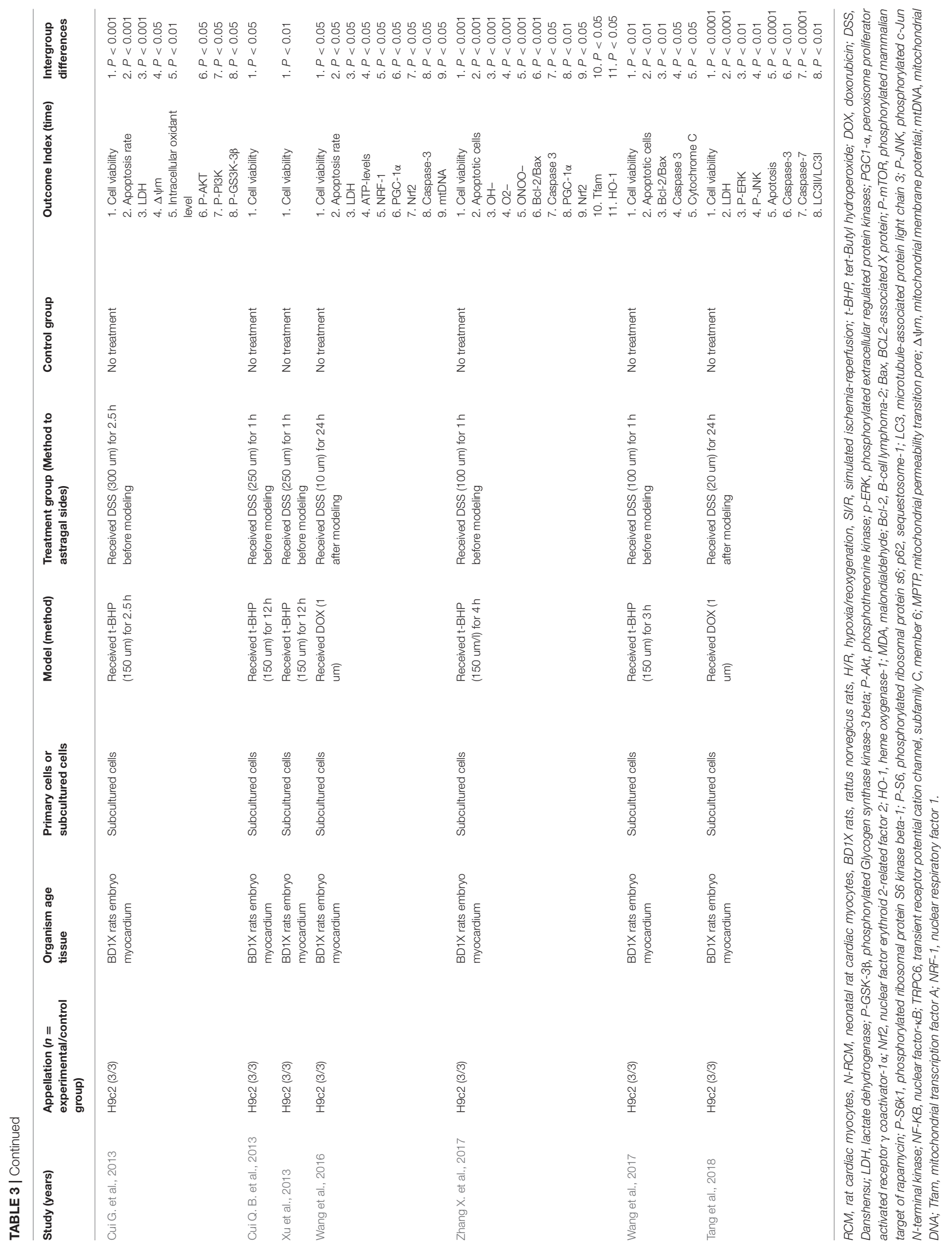


TABLE 4 | Risk of bias of the included in vivo studies.

\begin{tabular}{|c|c|c|c|c|c|c|c|c|c|c|c|}
\hline Study & A & B & C & D & $\mathbf{E}$ & $\mathbf{F}$ & G & $\mathbf{H}$ & I & $\mathbf{J}$ & Total \\
\hline Jiang et al., 1982 & $\sqrt{ }$ & & $\sqrt{ }$ & & & $\sqrt{ }$ & & & & & 3 \\
\hline Tang et al., 1989 & $\sqrt{ }$ & $\sqrt{ }$ & & & & $\sqrt{ }$ & & & & & 3 \\
\hline Li et al., 1996 & $\sqrt{ }$ & $\sqrt{ }$ & & & & $\sqrt{ }$ & & & & & 3 \\
\hline Zhang et al., 2010 & $\sqrt{ }$ & $\sqrt{ }$ & $\sqrt{ }$ & & & $\sqrt{ }$ & & & & $\sqrt{ }$ & 5 \\
\hline Lu et al., 2010 & $\sqrt{ }$ & & $\sqrt{ }$ & & & $\sqrt{ }$ & & & & & 3 \\
\hline Li et al., 2012 & $\sqrt{ }$ & $\sqrt{ }$ & $\sqrt{ }$ & & & $\sqrt{ }$ & & & $\sqrt{ }$ & $\sqrt{ }$ & 6 \\
\hline Yin et al., 2013 & $\sqrt{ }$ & $\sqrt{ }$ & $\sqrt{ }$ & & & $\sqrt{ }$ & & & $\sqrt{ }$ & $\sqrt{ }$ & 6 \\
\hline Yu J. et al., 2015 & $\sqrt{ }$ & $\sqrt{ }$ & $\sqrt{ }$ & & & $\sqrt{ }$ & & & $\sqrt{ }$ & $\sqrt{ }$ & 6 \\
\hline Wei et al., 2016 & $\sqrt{ }$ & $\sqrt{ }$ & $\sqrt{ }$ & & & $\sqrt{ }$ & & & & & 4 \\
\hline Song et al., 2016 & $\sqrt{ }$ & $\sqrt{ }$ & $\sqrt{ }$ & & & $\sqrt{ }$ & & & $\sqrt{ }$ & $\sqrt{ }$ & 6 \\
\hline Fan et al., 2016 & $\sqrt{ }$ & $\sqrt{ }$ & $\sqrt{ }$ & & & $\sqrt{ }$ & & & $\sqrt{ }$ & $\sqrt{ }$ & 6 \\
\hline Li et al., 2016 & $\sqrt{ }$ & $\sqrt{ }$ & $\sqrt{ }$ & & & $\sqrt{ }$ & & & $\sqrt{ }$ & $\sqrt{ }$ & 6 \\
\hline Hu et al., 2016 & $\sqrt{ }$ & $\sqrt{ }$ & $\sqrt{ }$ & & & $\sqrt{ }$ & & & $\sqrt{ }$ & $\sqrt{ }$ & 6 \\
\hline Yin et al., 2017 & $\sqrt{ }$ & $\sqrt{ }$ & $\sqrt{ }$ & $\sqrt{ }$ & & $\sqrt{ }$ & & & $\sqrt{ }$ & $\sqrt{ }$ & 7 \\
\hline Gao et al., 2017 & $\sqrt{ }$ & $\sqrt{ }$ & $\sqrt{ }$ & & & $\sqrt{ }$ & & & $\sqrt{ }$ & $\sqrt{ }$ & 6 \\
\hline Dong et al., 2009 & $\sqrt{ }$ & $\sqrt{ }$ & $\sqrt{ }$ & & & $\sqrt{ }$ & & & & $\sqrt{ }$ & 5 \\
\hline Xiang et al., 2009 & $\sqrt{ }$ & $\sqrt{ }$ & $\sqrt{ }$ & & & $\sqrt{ }$ & & & & & 4 \\
\hline Cheng et al., 2010 & $\sqrt{ }$ & $\sqrt{ }$ & $\sqrt{ }$ & & & $\sqrt{ }$ & & & & & 4 \\
\hline Zhao et al., 2012 & $\sqrt{ }$ & $\sqrt{ }$ & $\sqrt{ }$ & & & $\sqrt{ }$ & & & & & 4 \\
\hline Quan et al., 2012 & $\sqrt{ }$ & $\sqrt{ }$ & $\sqrt{ }$ & & & $\sqrt{ }$ & & & & & 4 \\
\hline Cui G. et al., 2013 & $\sqrt{ }$ & $\sqrt{ }$ & $\sqrt{ }$ & & & $\sqrt{ }$ & & & $\sqrt{ }$ & $\sqrt{ }$ & 6 \\
\hline Chen et al., 2014 & $\sqrt{ }$ & $\sqrt{ }$ & $\sqrt{ }$ & & & $\sqrt{ }$ & & & & & 4 \\
\hline Cui et al., 2014 & $\sqrt{ }$ & $\sqrt{ }$ & $\sqrt{ }$ & & & $\sqrt{ }$ & & & & $\sqrt{ }$ & 5 \\
\hline Wang et al., 2016 & $\sqrt{ }$ & $\sqrt{ }$ & & & & $\sqrt{ }$ & & & $\sqrt{ }$ & $\sqrt{ }$ & 5 \\
\hline $\begin{array}{l}\text { Zhang X. et al., } \\
2017\end{array}$ & $\sqrt{ }$ & & $\sqrt{ }$ & & & $\sqrt{ }$ & & & & $\sqrt{ }$ & 4 \\
\hline Wang et al., 2017 & $\sqrt{ }$ & $\sqrt{ }$ & $\sqrt{ }$ & & & $\sqrt{ }$ & & & $\sqrt{ }$ & $\sqrt{ }$ & 6 \\
\hline Tang et al., 2018 & $\sqrt{ }$ & $\sqrt{ }$ & & & & $\sqrt{ }$ & & & $\sqrt{ }$ & $\sqrt{ }$ & 5 \\
\hline
\end{tabular}

Studies fulfilling the criteria of: $A$, peer reviewed publication; $B$, control of temperature; $C$, random allocation to treatment or control; $D$, blinded induction of model; $E$, blinded assessment of outcome; $F$, use of anesthetic without significant intrinsic vascular protection activity; G, appropriate animal model (aged, diabetic, or hypertensive); $\mathrm{H}$, sample size calculation; I, compliance with animal welfare regulations; J, statement of potential conflict of interests.

$P=0.07, I^{2}=48 \%$; Figure 2A). Meta-analysis of five studies (Dong et al., 2009; Lu et al., 2010; Li et al., 2012; Wei et al., 2016; Yin et al., 2017) showed significant effects of DSS for reducing MI size than that of controls according to rat models of MI $(n=68$, SMD: $-1.81,95 \% \mathrm{CI}:-2.41$ to $-1.21, P<0.00001$; heterogeneity $\chi^{2}=1.05, P=0.90, I^{2}=0 \%$; Figure 2A). Moreover, one study (Jiang et al., 1982) showed positive effect of DSS on reducing MI size in canines $(P<0.05)$.

\section{Biochemical Markers of MI}

Meta-analysis of 8 studies (Dong et al., 2009; Xiang et al., 2009; Lu et al., 2010; Li et al., 2012; Cui et al., 2014; Yu J. et al., 2015; Fan et al., 2016; Song et al., 2016) showed DSS has significant effects on reducing LDH compared with controls $(n=116$, SMD: $-2.81,95 \%$ CI: -3.44 to $-2.18, P<0.00001$; heterogeneity $\chi^{2}$ $=35.07, P<0.0001, I^{2}=80 \%$ ). Two studies (Dong et al., 2009; Li et al., 2012) were removed through sensitivity analysis for simultaneous application of pretreatment and post-injury treatment. The remain six studies (Xiang et al., 2009; Lu et al., 2010; Cui et al., 2014; Yu J. et al., 2015; Fan et al., 2016; Song et al., 2016) showed significant effects on decreasing LDH in analysis $(n=88$, SMD: -2.58 , 95\% CI: -3.22 to $-1.95, P<$
TABLE 5 | Risk of bias of the included in vitro studies.

\begin{tabular}{|c|c|c|c|c|c|c|c|c|c|c|c|}
\hline Study & A & B & C & D & $\mathbf{E}$ & $\mathbf{F}$ & G & $\mathbf{H}$ & $\mathbf{I}$ & $\mathbf{J}$ & Total \\
\hline Zhu et al., 1999 & $\sqrt{ }$ & $\sqrt{ }$ & & & $\sqrt{ }$ & & & & & & 3 \\
\hline Guo et al., 2006 & $\sqrt{ }$ & & $\sqrt{ }$ & & $\sqrt{ }$ & & & & & & 3 \\
\hline Yin et al., 2013 & $\sqrt{ }$ & & $\sqrt{ }$ & & $\sqrt{ }$ & $\sqrt{ }$ & $\sqrt{ }$ & & & $\sqrt{ }$ & 6 \\
\hline Meng et al., 2016 & $\sqrt{ }$ & & $\sqrt{ }$ & & $\sqrt{ }$ & & & & & $\sqrt{ }$ & 4 \\
\hline Fan et al., 2016 & $\sqrt{ }$ & & $\sqrt{ }$ & & $\sqrt{ }$ & & & & & $\sqrt{ }$ & 4 \\
\hline Hu et al., 2016 & $\sqrt{ }$ & & $\sqrt{ }$ & & $\sqrt{ }$ & $\sqrt{ }$ & & & & $\sqrt{ }$ & 5 \\
\hline Gao et al., 2017 & $\sqrt{ }$ & & & & $\sqrt{ }$ & & & & & $\sqrt{ }$ & 3 \\
\hline Dong et al., 2009 & $\sqrt{ }$ & & $\sqrt{ }$ & & $\sqrt{ }$ & & & & & $\sqrt{ }$ & 4 \\
\hline Zhao et al., 2012 & $\sqrt{ }$ & & & & $\sqrt{ }$ & $\sqrt{ }$ & & & & & 3 \\
\hline Cui G. et al., 2013 & $\sqrt{ }$ & & & $\sqrt{ }$ & $\sqrt{ }$ & & & & & $\sqrt{ }$ & 4 \\
\hline Cui G. et al., 2013 & $\sqrt{ }$ & & $\sqrt{ }$ & $\sqrt{ }$ & $\sqrt{ }$ & $\sqrt{ }$ & & & & $\sqrt{ }$ & 4 \\
\hline Xu et al., 2013 & $\sqrt{ }$ & & & & $\sqrt{ }$ & & & & & $\sqrt{ }$ & 3 \\
\hline Wang et al., 2016 & $\sqrt{ }$ & & & & $\sqrt{ }$ & & & & & $\sqrt{ }$ & 4 \\
\hline $\begin{array}{l}\text { Zhang X. et al., } \\
2017\end{array}$ & $\sqrt{ }$ & & & & $\sqrt{ }$ & & & & & $\sqrt{ }$ & 3 \\
\hline Wang et al., 2017 & $\sqrt{ }$ & & & & $\sqrt{ }$ & & & & & & 3 \\
\hline Tang et al., 2018 & $\sqrt{ }$ & & & $\sqrt{ }$ & $\sqrt{ }$ & & & & & $\sqrt{ }$ & 4 \\
\hline
\end{tabular}

Studies fulfilling the criteria of: A, peer reviewed publication; B, use primary adult cardiomyocytes to study; C, cell lines with reliable source or validated by appropriate methods; D, assess toxicity of treatment on cells; $E$, culture environment (culture media/sera, $\mathrm{pH} / \mathrm{CO}_{2}$, and temperature); $F$, random allocation to treatment or control; $G$, blinded induction of model; $\mathrm{H}$, blinded assessment of outcome; I, calculation of the sample size necessary to achieve sufficient power; J, statement of potential conflict of interest.

0.00001; heterogeneity $\chi^{2}=9.03, P=0.11, I^{2}=45 \%$; Figure $2 B$ ). Meta-analysis of seven studies (Dong et al., 2009; Xiang et al., 2009; Lu et al., 2010; Cui et al., 2014; Yu J. et al., 2015; Fan et al., 2016; Song et al., 2016) showed DSS has significant effects on decreasing CK compared with controls ( $n=100$, SMD: -3.16 , 95\% CI: -3.86 to $-2.46, P<0.00001$; heterogeneity $\chi^{2}=20.32$, $\left.P=0.002, I^{2}=70 \%\right)$. Through sensitivity analysis, we removed two studies which utilized ISO in modeling (Song et al., 2016) and the other with unknown administration route (Xiang et al., 2009). Meta-analysis of 5 studies (Dong et al., 2009; Lu et al., 2010; Cui et al., 2014; Yu J. et al., 2015; Fan et al., 2016) showed the significant effects on reducing $\mathrm{CK}(n=68$, SMD: $-2.68,95 \%$ CI: -3.42 to $-1.94, P<0.00001$; heterogeneity $\chi^{2}=6.27, P=$ $0.18, I^{2}=36 \%$; Figure 2C). Five studies (Li et al., 2012, 2016; Quan et al., 2012; Yin et al., 2013; Hu et al., 2016) failed to pool the analysis, but they all showed DSS has significant effects on decreasing $\mathrm{CK}-\mathrm{MB}$ compared with control $(P<0.05$, or $P<$ 0.01 , or $P<0.001$ ). One study (Li et al., 2016) showed DSS has significant effects on decreasing $\mathrm{CTnT}(P<0.001)$.

\section{The level of ST-segment elevation and/or LVEFand/or LVFS}

One study ( $\mathrm{Li}$ et al., 2012) showed DSS significantly inhibit the ST-segment elevation compared with control $(P<0.001)$. Anothe study (Yin et al., 2017) showed positive effects of DSS on improving LVEF and LVFS compared with control $(P<0.05)$.

\section{Cardioprotective mechanisms}

Comparing DSS with controls, meta-analysis of two studies (Cui G. et al., 2013; Li et al., 2016) showed significant effects on increasing phosphatidylinositol 3-kinase (p-PI3K) $(n=20$, SMD: 3.56, 95\% CI: 1.82-5.29, $P<0.0001$; heterogeneity $\chi^{2}=$ $0.96, P=0.33, I^{2}=0 \%$; Figure 3A); two studies (Yu J. et al., 


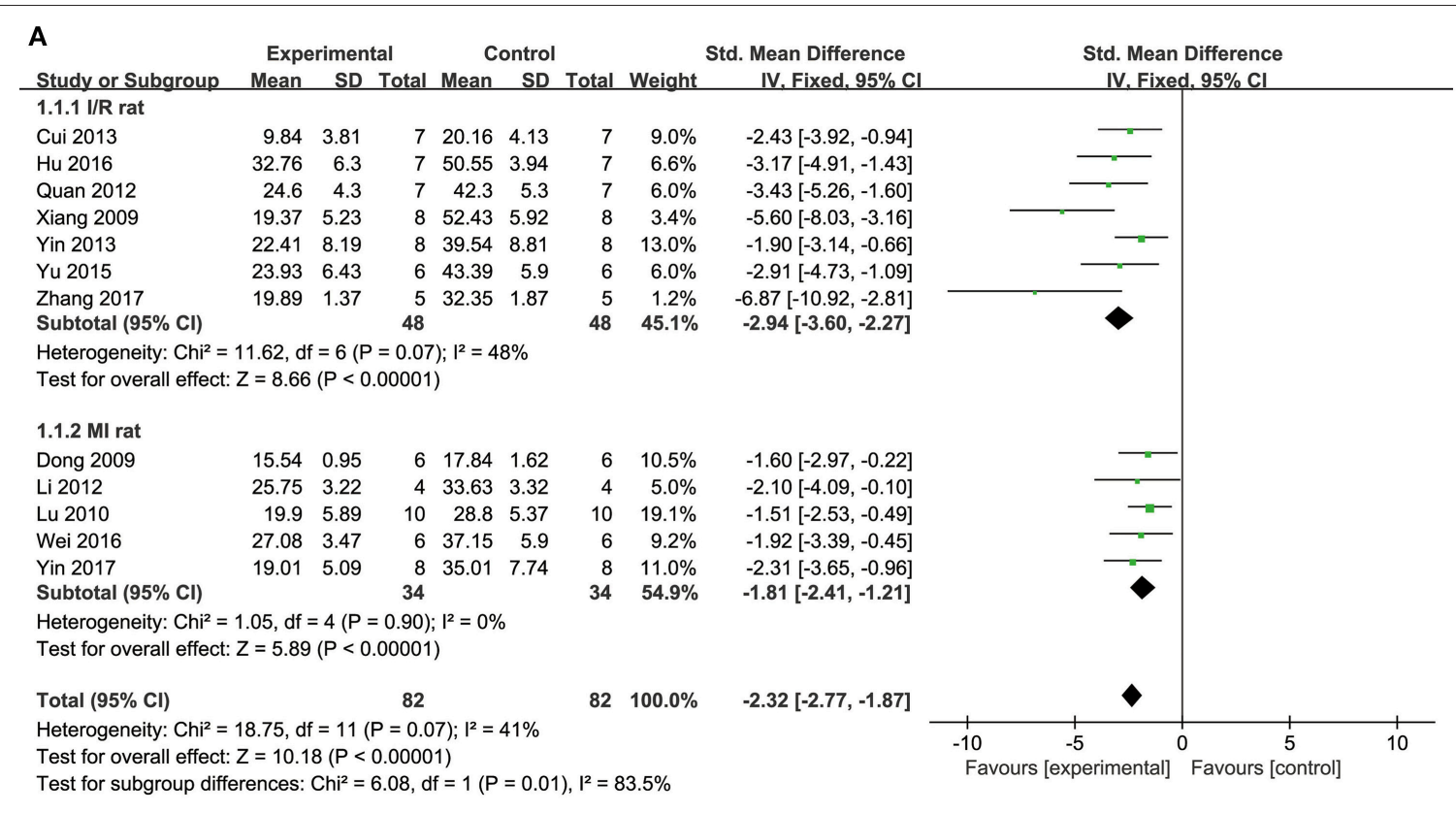

B

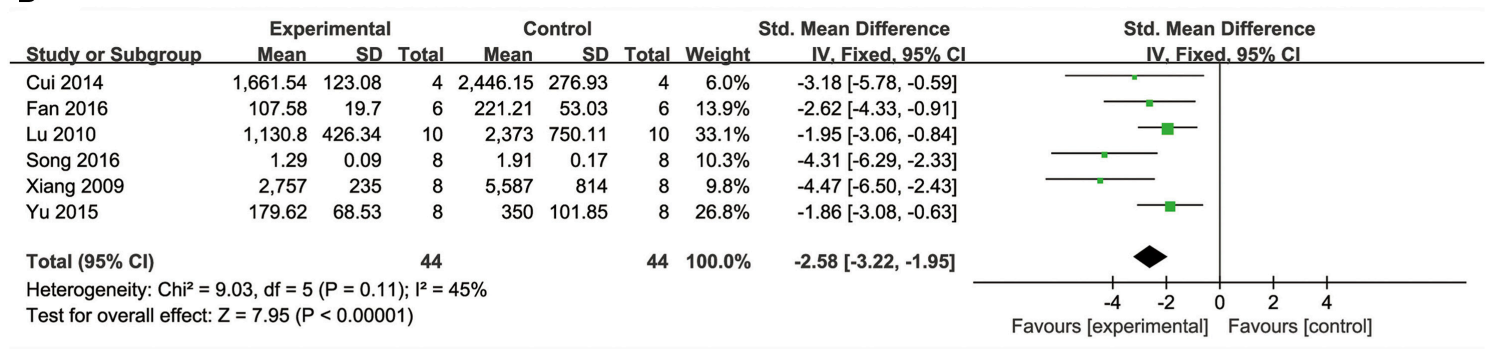

C

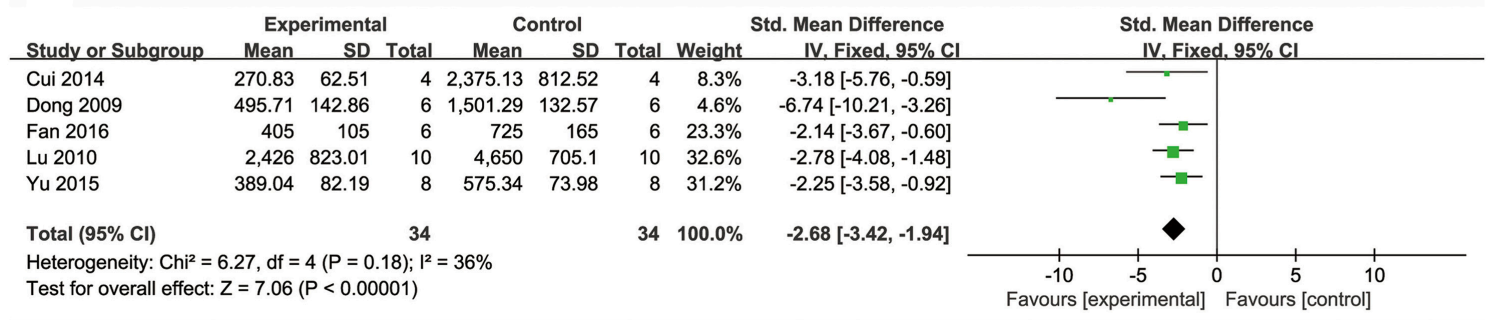

FIGURE 2 | The forest plot: (A) in vivo effects of danshensu on reducing myocardial infarction size compared with controls ( $n=82$ per group); (B) in vivo effects of danshensu on reducing LDH compared with controls ( $n=44$ per group); (C) in vivo effects of danshensu on reducing CK compared with controls $(n=34$ per group). $\mathrm{LDH}$, lactate dehydrogenase; CK, creatine kinase.

2015; Li et al., 2016) for increasing phosphothreonine kinase (p-Akt) $(n=18$, SMD: 14.22, 95\% CI: 7.84-20.60, $P<0.0001$; heterogeneity $\chi^{2}=0.73, P=0.39, I^{2}=0 \%$; Figure $3 \mathrm{~B}$ ); four studies ( $\mathrm{Li}$ et al., 2012, 2016; Cui G. et al., 2013; Yu J. et al., 2015) for increasing nuclear factor erythroid 2-related factor 2 (Nrf2) ( $n=42$, SMD: 2.42, 95\% CI: 1.50-3.33, $P<0.00001$; heterogeneity $\chi^{2}=2.05, P=0.56, I^{2}=0 \%$; Figure $\left.3 \mathrm{C}\right) ; 2$ studies (Cui G. et al., 2013; Li et al., 2016) for increasing heme oxygenase-1 (HO- 1$)$ ( $n=20$, SMD: 2.68 , 95\% CI: 1.21-4.15, $P=0.0003$; heterogeneity $\chi^{2}=1.41, P=0.24, I^{2}=29 \%$;
Figure 3D); two studies ( $\mathrm{Li}$ et al., 2012, 2016) for increasing glutathione-S-transferase (GST) $(n=32$, SMD: 3.35 , 95\% CI: 2.16-4.54, $P<0.00001$; heterogeneity $\chi^{2}=1.47, P=0.22, I^{2}$ $=32 \%$; Figure 3E); two studies (Tang et al., 1989; Yu J. et al., 2015) for increasing Glutathione peroxidase (GSH-Px) $(n=24$, SMD: 3.22, 95\% CI: 1.84-4.60, $P<0.00001$; heterogeneity $\chi^{2}=$ $0.49, P=0.48, I^{2}=0 \%$; Figure 3F); two studies (Li et al., 2012, 2016) for increasing glutathione synthetase (GSH) $(P<0.001)$; three studies (Cui et al., 2014; Yu J. et al., 2015; Hu et al., 2016) for increasing superoxide dismutase (SOD) $(n=38$, SMD: 3.10 , 


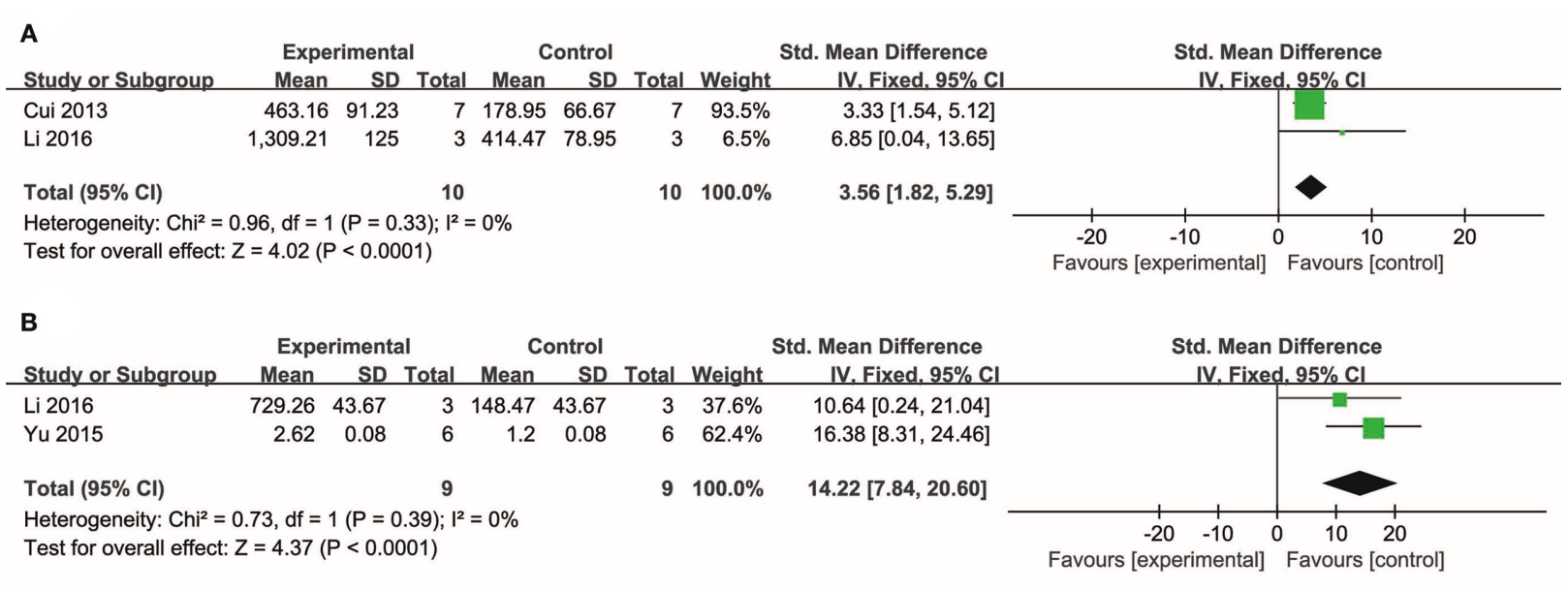

C

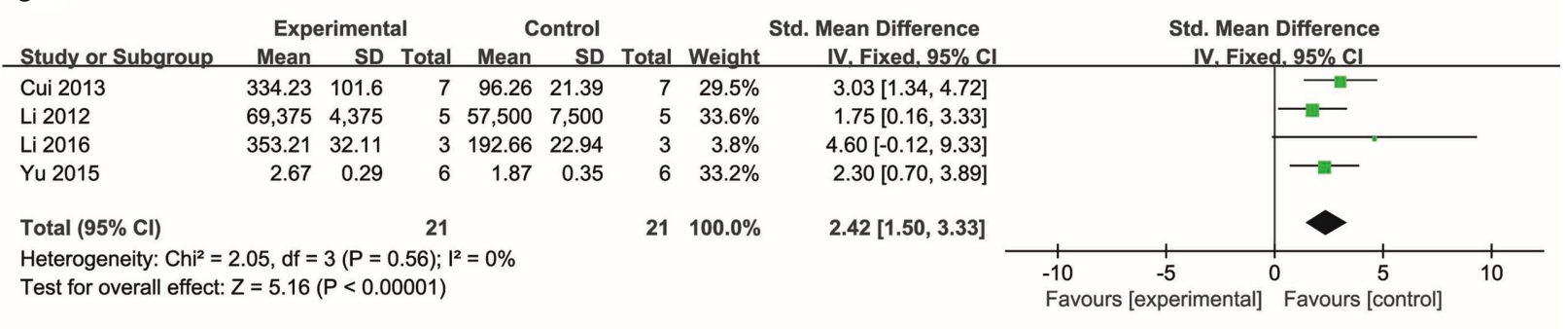

D

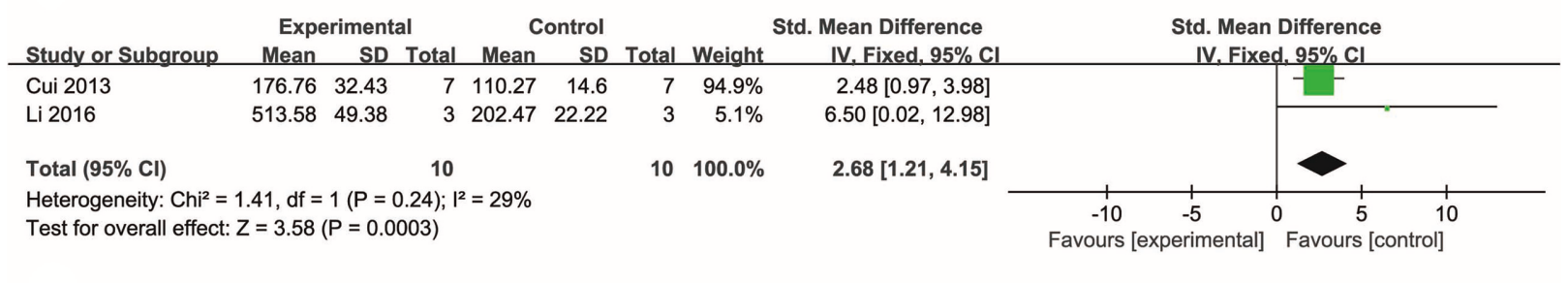

E

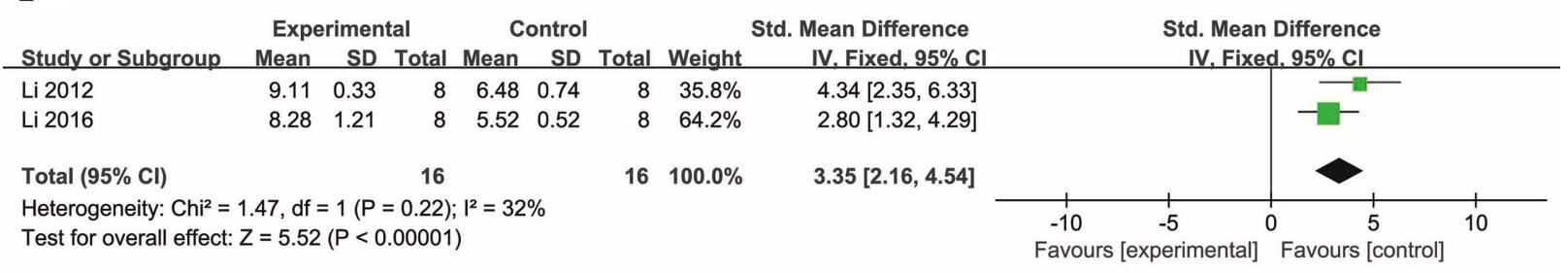

$\mathbf{F}$

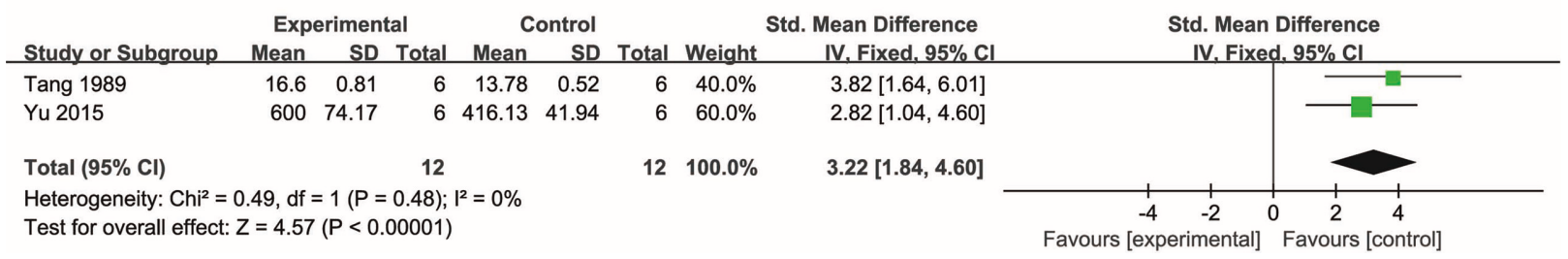

FIGURE 3 | The forest plot: (A) in vivo effects of danshensu for increasing p-PI3K compared with controls ( $n=10$ per group); (B) in vivo effects of danshensu for increasing p-AKT compared with controls ( $n=9$ per group); (C) in vivo effects of danshensu for increasing Nrf2 compared with controls ( $n=21$ per group); (D) in vivo effects of danshensu for increasing HO-1compared with controls ( $n=10$ per group); (E) in vivo effects of danshensu for increasing GST compared with controls ( $n=$ 16 per group); (F) in vivo effects of danshensu for increasing GSH-Pxcompared with controls ( $n=12$ per group). p-PI3K, phosphatidylinositol 3-kinase; p-AKT, phosphothreonine kinase; Nrf2, nuclear factor erythroid 2-related factor 2; HO-1, heme oxygenase-1; GST, glutathione-S-transferase; GSH-Px, glutathione peroxidase. 

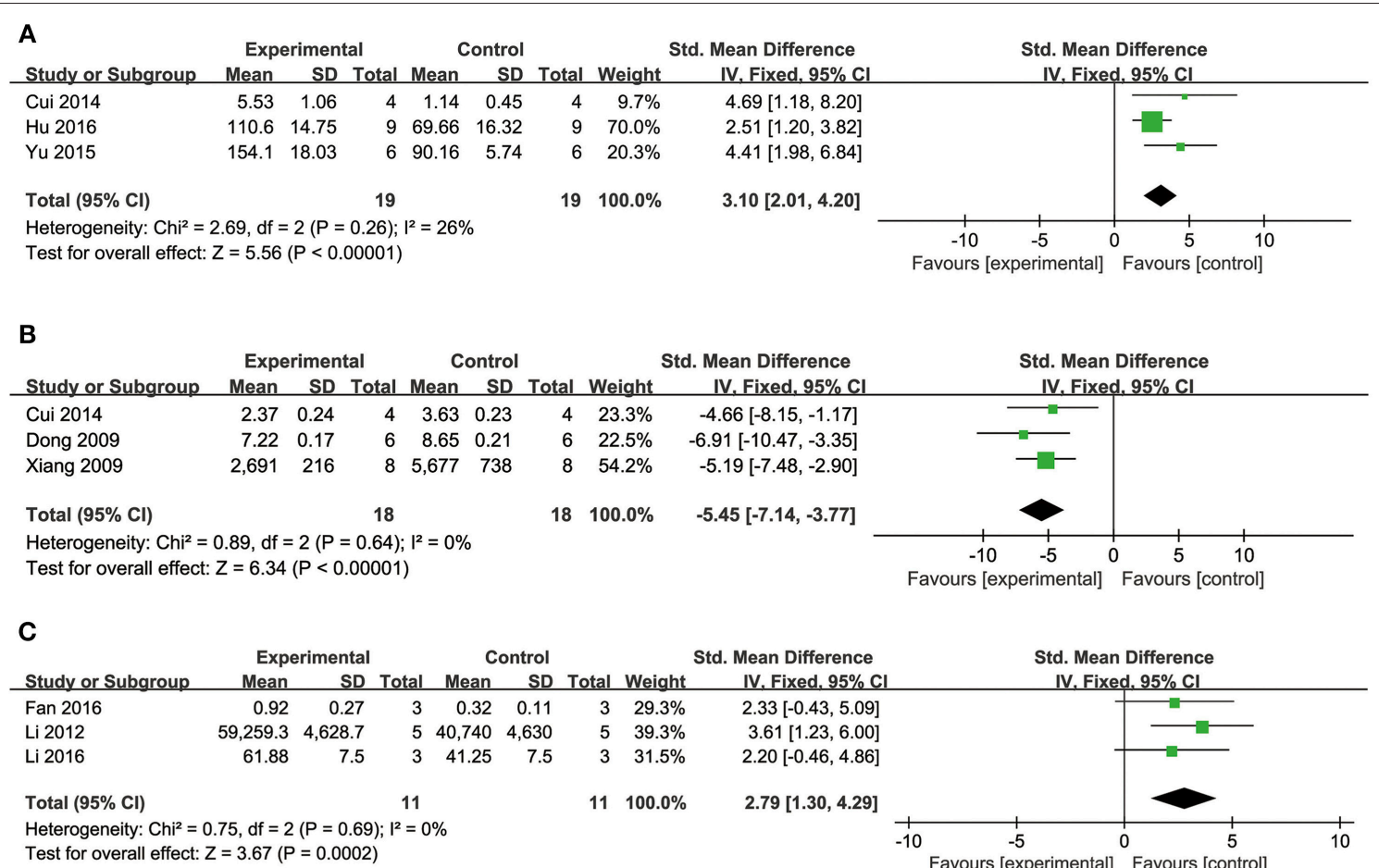

\begin{tabular}{|c|c|c|c|c|c|c|c|c|}
\hline \multirow[b]{2}{*}{ Study or Subgroup } & \multicolumn{3}{|c|}{ Experimental } & \multicolumn{3}{|c|}{ Control } & \multicolumn{2}{|r|}{ Std. Mean Difference } \\
\hline & Mean & SD & Total & Mean & SD & Total & Weight & IV. Fixed, $95 \% \mathrm{Cl}$ \\
\hline Fan 2016 & 1.59 & 0.43 & 3 & 3.1 & 0.46 & 3 & $28.2 \%$ & $-2.71[-5.78,0.36]$ \\
\hline Li 2012 & 71,296 & 5,555 & 5 & 98,148 & 10,185 & 5 & $61.7 \%$ & $-2.96[-5.03,-0.88]$ \\
\hline Li 2016 & 256.79 & 29.63 & 3 & 553.08 & 59.26 & 3 & $10.1 \%$ & $-5.06[-10.20,0.08]$ \\
\hline Total $(95 \% \mathrm{Cl})$ & & & 11 & & & 11 & $100.0 \%$ & $-3.10[-4.73,-1.47]$ \\
\hline
\end{tabular}

Heterogeneity: $\mathrm{Chi}^{2}=0.64, \mathrm{df}=2(\mathrm{P}=0.73) ; \mathrm{I}^{2}=0 \%$ Test for overall effect: $Z=3.73(P=0.0002)$

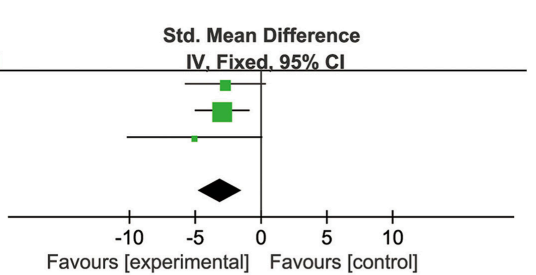

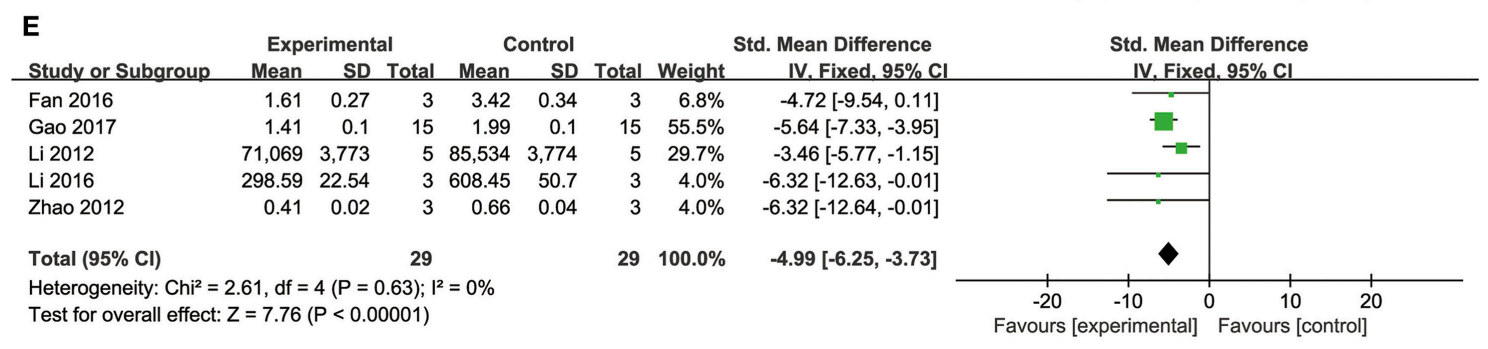

FIGURE 4 | The forest plot: (A) in vivo effects of danshensu for increasing SOD compared with controls ( $n=19$ per group); (B) in vivo effects of danshensu for reducing MDA compared with controls ( $n=18$ per group); (C) in vivo effects of danshensu for increasing Bcl-2 compared with controls $(n=11$ per group); (D) in vivo effects of danshensu for reducing Bax compared with controls ( $n=11$ per group); (E) in vivo effects of danshensu for reducing caspase-3 compared with controls ( $n$ = 29 per group). SOD, superoxide dismutase; MDA, malondialdehyde; Bcl-2, B-cell lymphoma-2; Bax, BCL2-associated X protein.

95\% CI: 2.10-4.20, $P<0.00001$; heterogeneity $\chi^{2}=2.69, P$ $=0.26, I^{2}=26 \%$; Figure 4A); three studies(Dong et al., 2009; Xiang et al., 2009; Cui et al., 2014) for reducing malondialdehyde (MDA) $(n=36$, SMD: $-5.45,95 \% \mathrm{CI}:-7.14$ to $-3.77, P<$ 0.00001 ; heterogeneity $\chi^{2}=0.89, P=0.64, I^{2}=0 \%$; Figure 4B); three studies (Li et al., 2012, 2016; Fan et al., 2016) for increasing B-cell lymphoma-2 (Bcl-2) ( $n=22$, SMD: 2.79 , 95\% CI: $1.30-$ 4.29, $P=0.0002$; heterogeneity $\chi^{2}=0.75, P=0.69, I^{2}=0 \%$; Figure 4C); three studies (Li et al., 2012, 2016; Fan et al., 2016) for reducing BCL2-associated X protein $(\operatorname{Bax})(n=22, \mathrm{SMD}$ : $-3.10,95 \%$ CI: -4.73 to $-1.47, P=0.0002$; heterogeneity $\chi^{2}$
$=0.64, P=0.73, I^{2}=0 \%$; Figure 4D); one study (Zhao et al., 2012) for reducing $\mathrm{Bcl} 2 / \mathrm{Bax}(P<0.01)$; five studies (Li et al., 2012, 2016; Zhao et al., 2012; Fan et al., 2016; Gao et al., 2017) for reducing caspase-3 $(n=58$, SMD: $-4.99,95 \% \mathrm{CI}:-6.25$ to $-3.73, P<0.00001$; heterogeneity $\chi^{2}=2.61, P=0.63, I^{2}=$ $0 \%$; Figure 4E); two studies (Quan et al., 2012; Li et al., 2016) for reducing tumor necrosis factor- $\alpha$ (TNF- $\alpha)(n=20$, SMD: $-2.40,95 \% \mathrm{CI}:-3.71$ to $-1.10, P=0.0003$; heterogeneity $\chi^{2}$ $=0.11, P=0.74, I^{2}=0 \%$; Figure 5A); one study (Quan et al., 2012) for reducing Interleukin-1 (IL-1) and Interleukin-6 (IL-6) $(P<0.05)$; two studies (Wei et al., 2016; Yin et al., 2017) for 

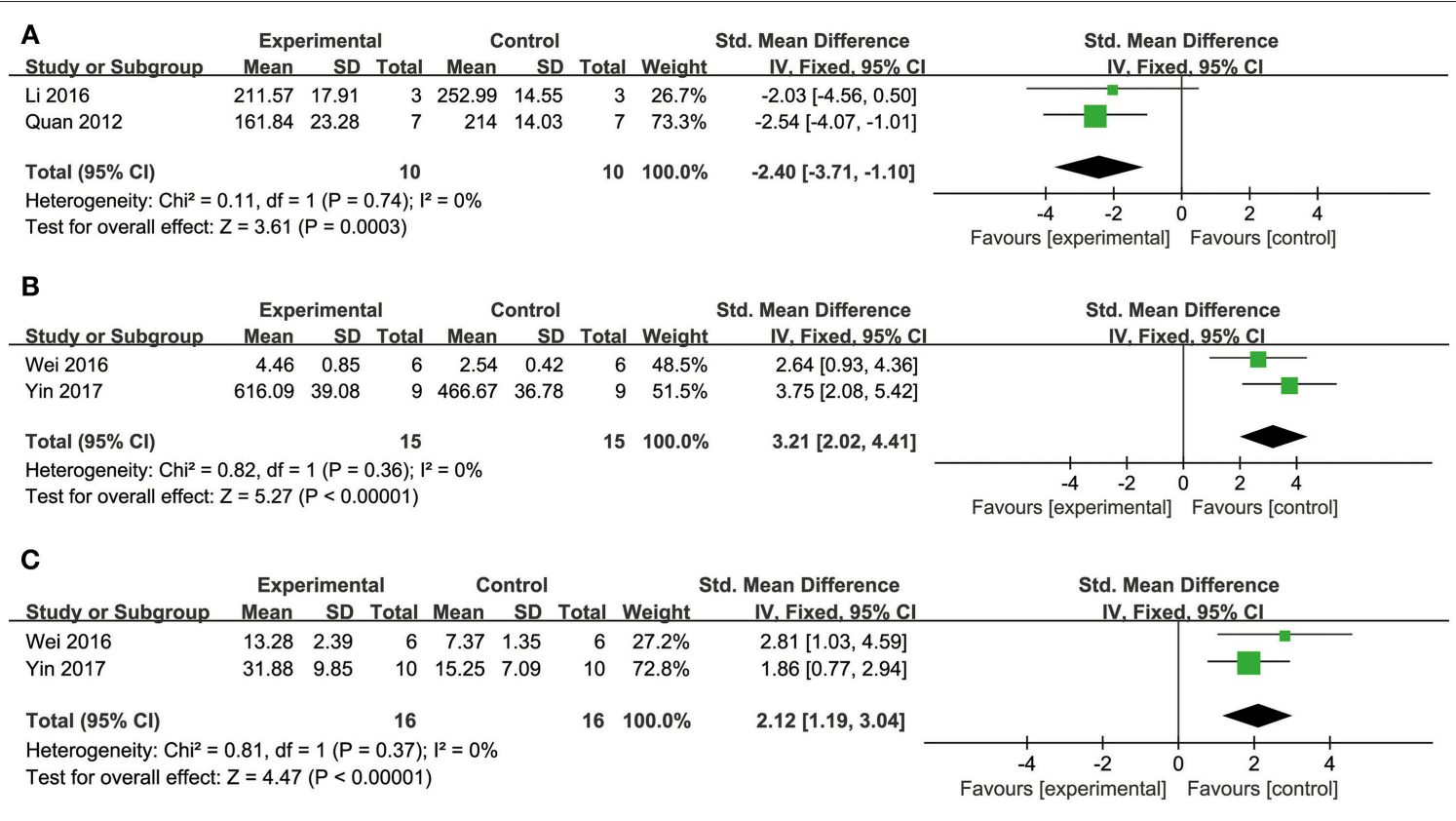

D

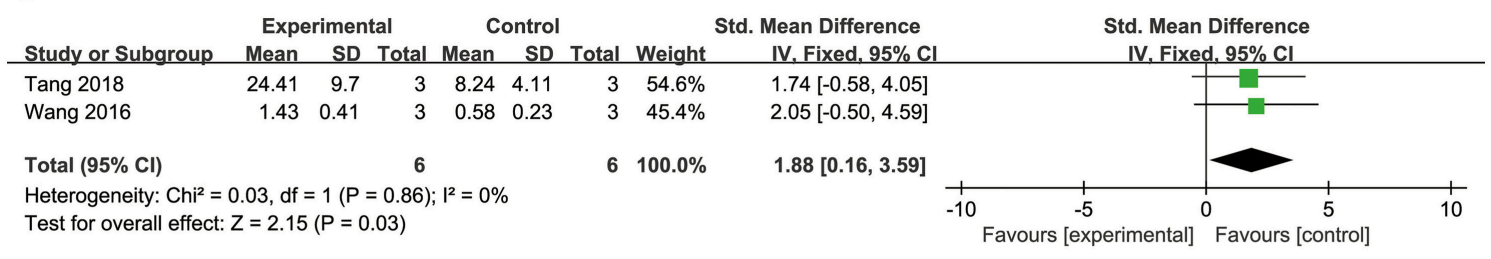

E

\begin{tabular}{|c|c|c|c|c|c|c|c|c|c|}
\hline \multirow[b]{2}{*}{ Study or Subgroup } & \multicolumn{3}{|c|}{ Experimental } & \multicolumn{2}{|c|}{ Control } & \multirow{2}{*}{ Total } & \multicolumn{2}{|c|}{ Std. Mean Difference } & \multirow{2}{*}{$\begin{array}{l}\text { Std. Mean Difference } \\
\text { IV. Fixed. } 95 \% \mathrm{Cl}\end{array}$} \\
\hline & Mean & SD & Total & Mean & SD & & Weight & IV, Fixed, $95 \% \mathrm{Cl}$ & \\
\hline Tang 2018 & 35.83 & 15.67 & 3 & 9.17 & 5 & 3 & $59.3 \%$ & $1.83[-0.55,4.22]$ & \\
\hline Wang 2016 & 28.16 & 8.9 & 3 & 7.35 & 3.26 & 3 & $40.7 \%$ & $2.48[-0.40,5.37]$ & \\
\hline Total $(95 \% \mathrm{Cl})$ & & & 6 & & & 6 & $100.0 \%$ & $2.10[0.26,3.94]$ & \\
\hline \multicolumn{9}{|c|}{$\begin{array}{l}\text { Heterogeneity: } \mathrm{Chi}^{2}=0.12, \mathrm{df}=1(P=0.73) ; \mathrm{I}^{2}=0 \% \\
\text { Test for overall effect: } Z=2.24(P=0.03)\end{array}$} & $\begin{array}{ccccc}-10 & -5 & 0 & 5 & 10 \\
\text { Favours [experimental] } & \text { Favours [control] }\end{array}$ \\
\hline
\end{tabular}

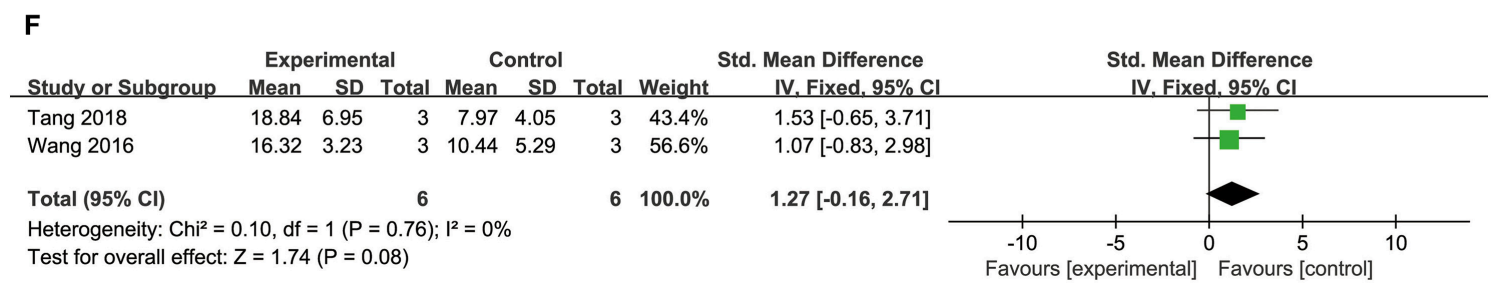

FIGURE 5 | The forest plot: (A) in vivo effects of danshensu for reducing TNF- $\alpha$ compared with controls ( $n=10$ per group); (B) in vivo effects of danshensu for increasing SDF-1 compared with controls ( $n=15$ per group); (C) in vivo effects of danshensu for increasing MVD compared with controls ( $n=16$ per group); (D) in vivo effects of danshensu for increasing stroke volume compared with controls ( $n=6$ per group); (E) in vivo effects of danshensu for increasing cardiac output compared with controls ( $n=6$ per group); (F) in vivo effects of danshensu for increasing fractional shortening compared with controls $(n=6$ per group). TNF- $\alpha$, tumor necrosis factor- $\alpha$; SDF-1, stromal cell-derived factor-1 $\alpha$; MVD, micro vessel density.

increasing stromal cell-derived factor- $1 \alpha($ SDF- 1$)(n=30$, SMD: 3.21, 95\% CI: 2.02-4.41, $P<0.00001$; heterogeneity $\chi^{2}=0.82$, $P=0.36, I^{2}=0 \%$; Figure 5B), micro vessel density (MVD) ( $n$ $=32$, SMD: 2.12 , 95\% CI: 1.19-3.04, $P<0.00001$; heterogeneity $\chi^{2}=0.81, P=0.37, I^{2}=0 \%$; Figure 5C), C-X-C chemokine receptor type $4(\mathrm{CXCR}-4)(P<0.01)$ and vascular endothelial growth factor (VEGF) $(P<0.05)$; one study (Yin et al., 2017) for increasing basic fibroblast growth factor (bFGF) $(P<0.05)$; three studies (Li et al., 1996; Cheng et al., 2010; Zhang et al., 2010) for increasing adenosine triphosphate (ATP) $(P<0.01$ ORP $<0.05$ ORP $<0.001$ ); two studies (Li et al., 1996; Zhang et al., 2010) for increasingenergy charge(EC) $(P<0.01)$; one study 


\begin{tabular}{|c|c|c|c|c|c|}
\hline \multirow{2}{*}{$\begin{array}{l}\text { A } \\
\text { Study or Subgroup }\end{array}$} & \multicolumn{3}{|c|}{ Experimental } & \multicolumn{2}{|c|}{ Control } \\
\hline & Mean & SD & Total & Mean & SD \\
\hline Cui 2013 (1) & 85.23 & 10.55 & 4 & 12.24 & 1.69 \\
\hline Cui 2013 (2) & 91.14 & 18.59 & 3 & 52.56 & 1.92 \\
\hline Dong 2009 & 44.12 & 0.38 & 6 & 38.92 & 0.11 \\
\hline Fan 2016 & 49.47 & 1.58 & 3 & 33.68 & 2.1 \\
\hline Gao 2017 & 0.8 & 0.03 & 3 & 0.65 & 0.04 \\
\hline Hu 2016 & 69.44 & 3.7 & 6 & 35.26 & 6.1 \\
\hline Tang 2018 & 0.8 & 0.05 & 3 & 0.51 & 0.03 \\
\hline Wang 2016 & 90.06 & 13.04 & 3 & 54.66 & 4.97 \\
\hline Wang 2017 & 82.89 & 3.29 & 3 & 28.62 & 0.66 \\
\hline Xu 2013 & 101.31 & 3.94 & 3 & 38.36 & 11.31 \\
\hline Yin 2013 & 96.6 & 7.9 & 5 & 69.6 & 2.1 \\
\hline Zhang 2017 & 88.31 & 2.27 & 3 & 29.55 & 0.65 \\
\hline Total $(95 \% \mathrm{Cl})$ & & & 45 & & \\
\hline \multicolumn{6}{|c|}{ Heterogeneity: $\mathrm{Chi}^{2}=21.13, \mathrm{df}=11(\mathrm{P}=0.03) ; \mathrm{I}^{2}=48 \%$} \\
\hline
\end{tabular}

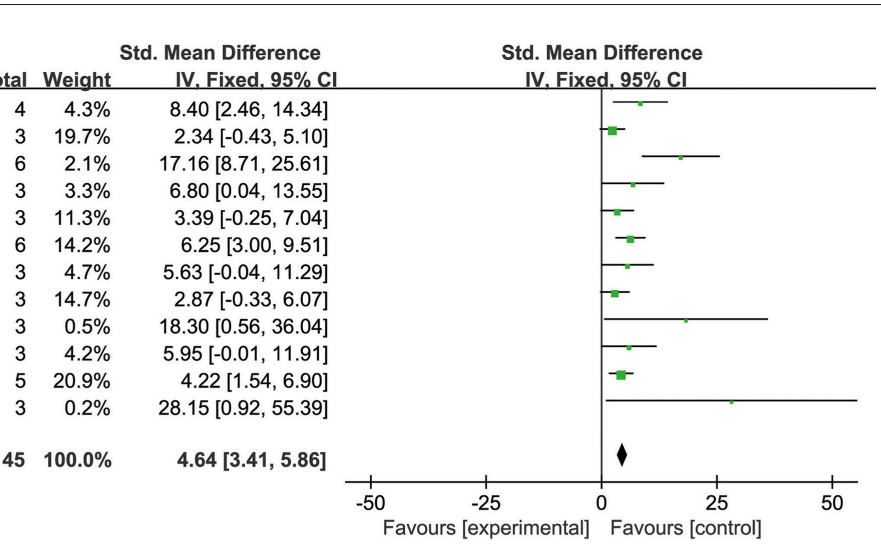

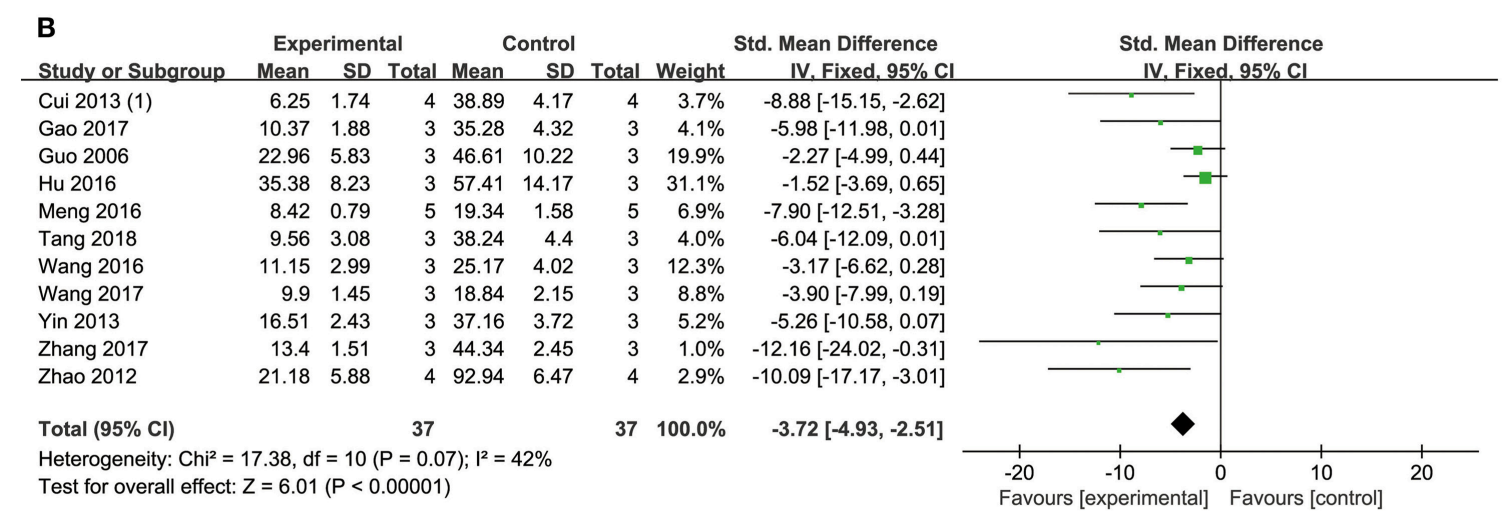
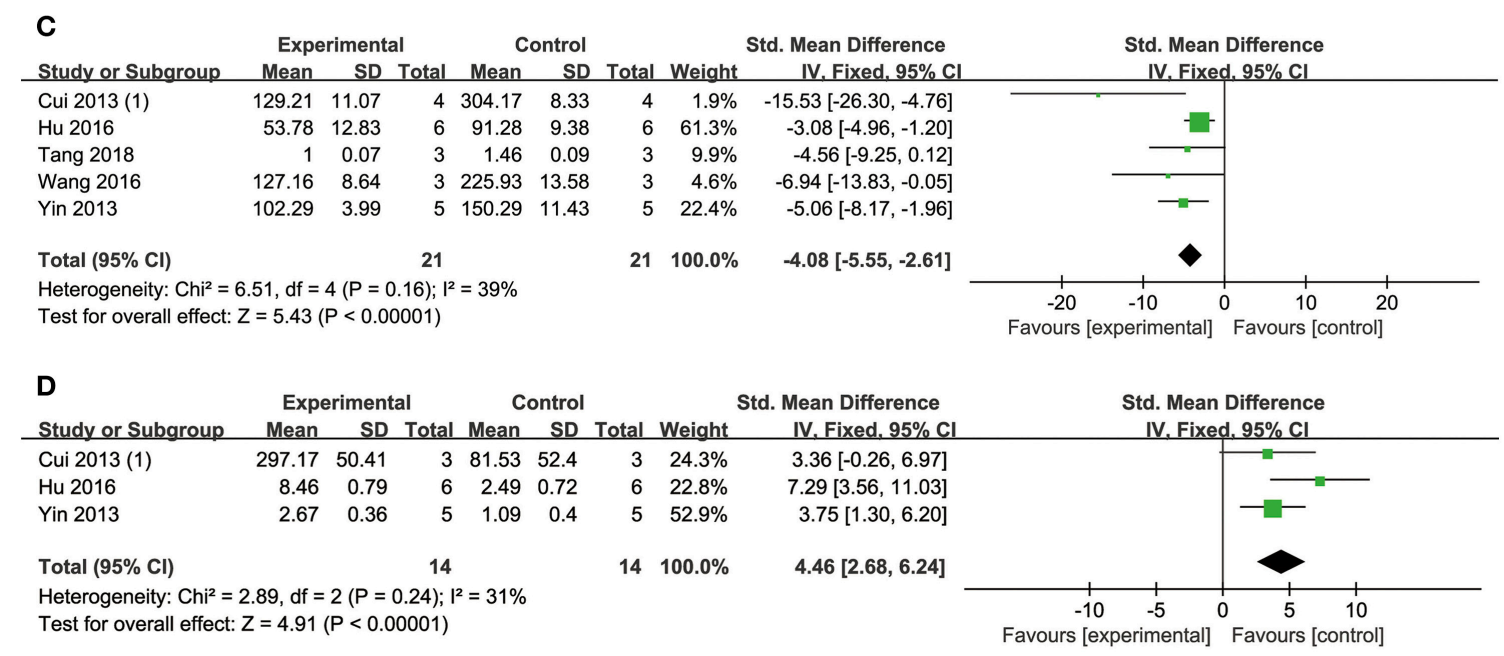

FIGURE 6 | The forest plot: (A) in vitro effects of danshensu for increasing cell viability compared with controls ( $n=45$ per group); (B) in vitro effects of danshensu for reducing apoptosis rate compared with controls ( $n=37$ per group); (C) in vitro effects of danshensu for reducing LDH compared with controls $(n=21$ per group); (D) in vitro effects of danshensu for increasing p-AKT compared with controls ( $n=14$ per group). LDH, lactate dehydrogenase; p-AKT, phosphothreonine kinase.

(Gao et al., 2017) for inhibiting Mitochondrial permeability transition pore (MPTP) opening $(P<0.05)$; one study (Fan et al., 2016) for increasing phosphorylated mammalian target of rapamycin (p-mTOR) $(P<0.05)$, two studies (Wang et al., 2016; Tang et al., 2018) for increasing stroke volum $(n=12$, SMD: $1.88,95 \%$ CI: $0.16-3.59, P=0.03$; heterogeneity $\chi^{2}$ $=0.03, P=0.86, I^{2}=0 \%$; Figure 5D), cardiac output $(n=$
12, SMD: 2.10, 95\% CI: $0.26-3.94, P=0.03$; heterogeneity $\chi^{2}=0.12, P=0.73, I^{2}=0 \%$; Figure 5E) and fractional shortening $(n=12$, SMD: $1.27,95 \% \mathrm{CI}:-0.16$ to $2.71, P=0.08$; heterogeneity $\chi^{2}=0.10, P=0.76, I^{2}=0 \%$; Figure $\left.5 \mathrm{~F}\right)$. We summarized a schematic representation of cardioprotective mechanism of DSS for myocardial ischemic injury (Figure 8). 


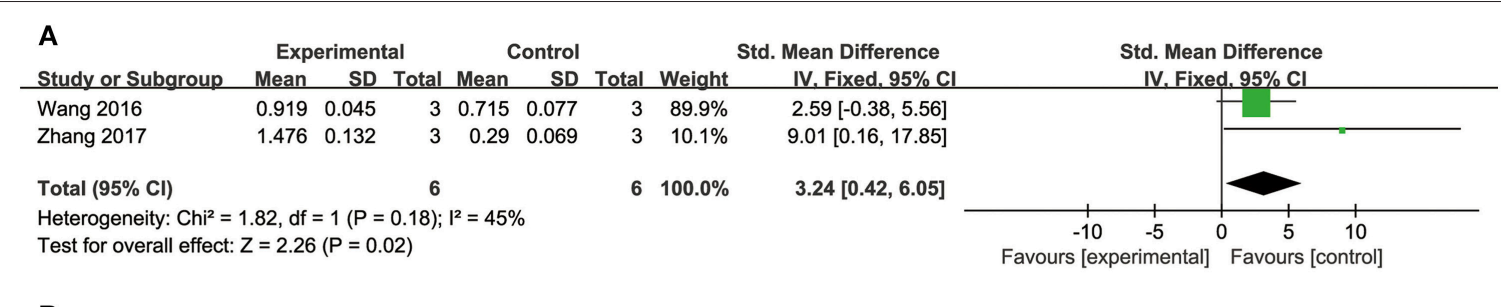

B

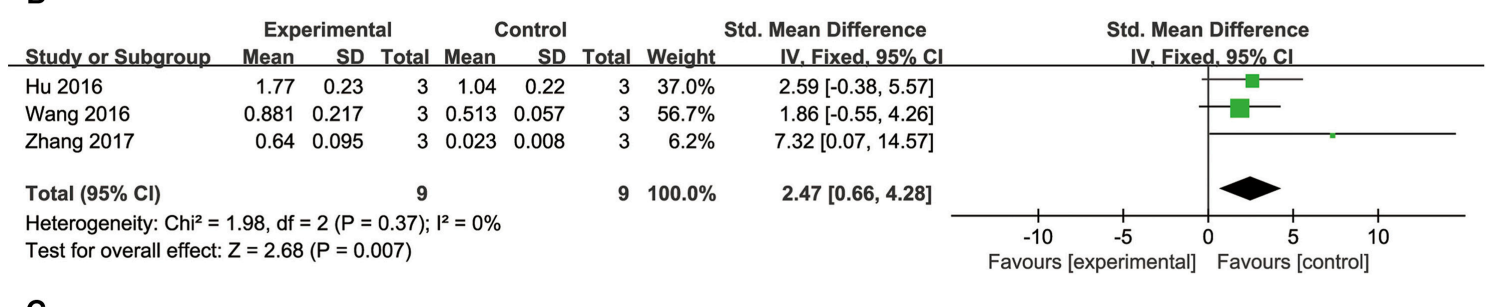

C

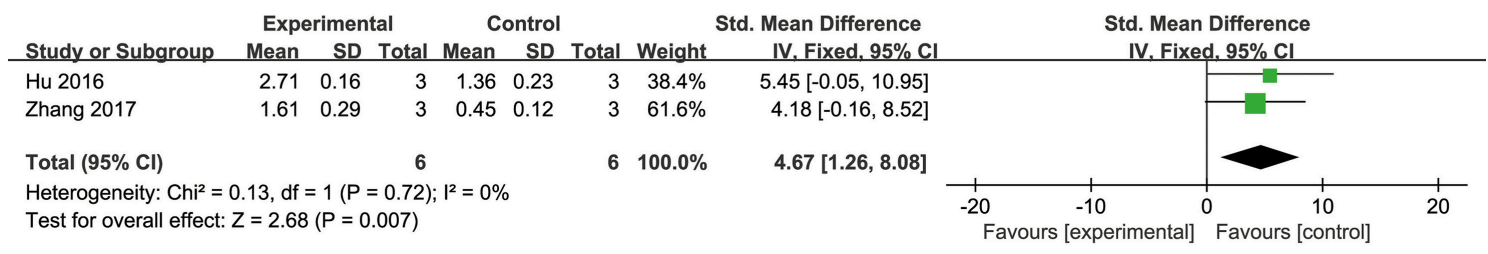

D

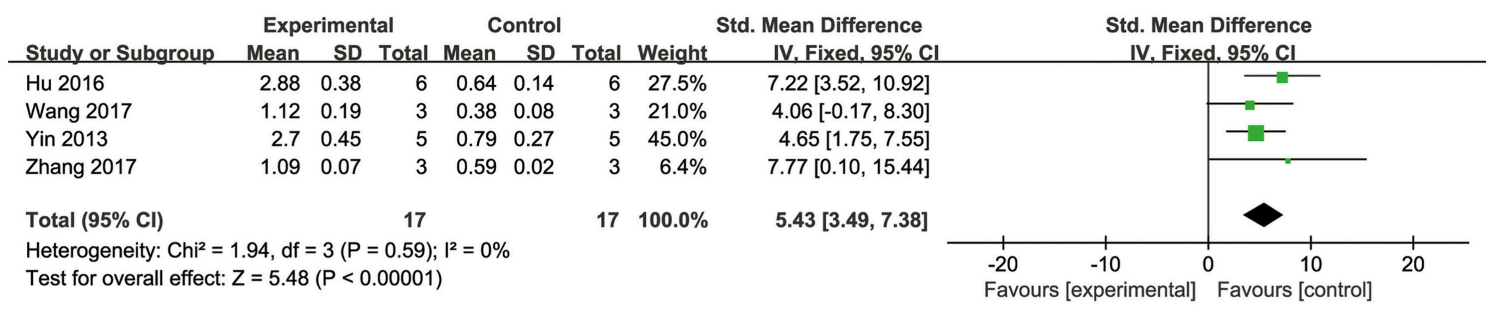

E

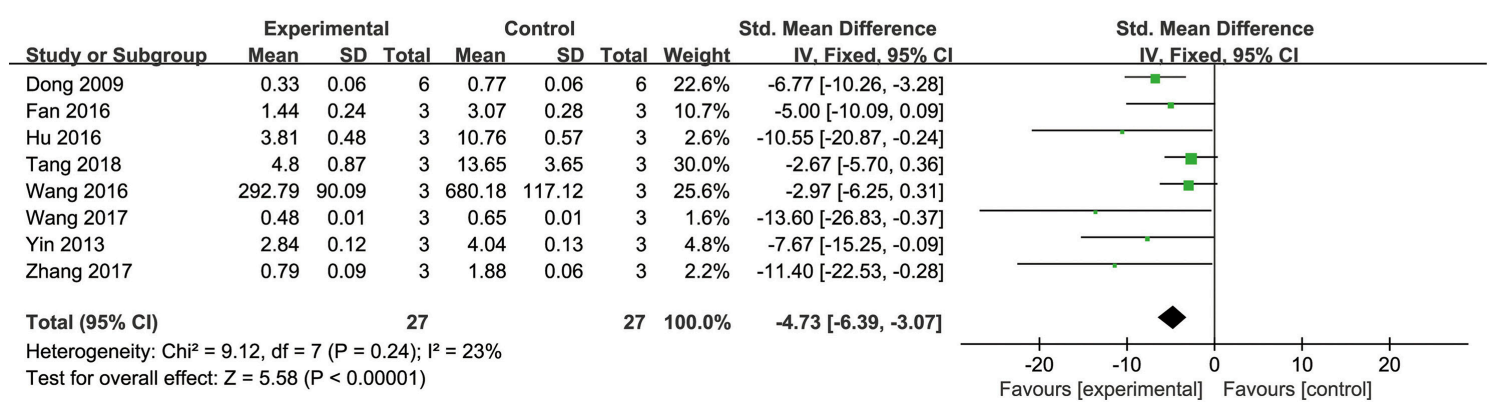

FIGURE 7 | The forest plot: (A) in vitro effects of danshensu for increasing PGC1- $\alpha$ compared with controls ( $n=6$ per group); (B) in vitro effects of danshensu for increasing Nrf2 compared with controls ( $n=9$ per group); (C) in vitro effects of danshensu for increasing HO-1 compared with controls ( $n=6$ per group); (D) in vitro effects of danshensu for increasing Bcl-2/Bax compared with controls ( $n=17$ per group); (E) in vitro effects of danshensu for reducing caspase-3 compared with controls ( $n=27$ per group). PGC1- $\alpha$, peroxisome proliferator activated receptor $\gamma$ coactivator-1 $\alpha$; Nrf2, nuclear factor erythroid 2-related factor 2; HO-1, heme oxygenase-1; Bcl-2/Bax, B-cell lymphoma-2/BCL2-associated X protein.

\section{Cell Viability and Apoptosis Rate Cell viability}

Meta-analysis of 12 studies (Dong et al., 2009; Cui G. et al., 2013; Cui Q. B. et al., 2013; Xu et al., 2013; Yin et al., 2013; Fan et al., 2016; Hu et al., 2016; Wang et al., 2016, 2017;
Gao et al., 2017; Zhang X. et al., 2017; Tang et al., 2018) showed DSS has significant effects on improving cell viability compared with controls ( $n=90$, SMD: 4.64, 95\% CI: 3.41-5.86, $P<0.00001$; heterogeneity $\chi^{2}=21.13, P=0.03, I^{2}=48 \%$; Figure 6A). 


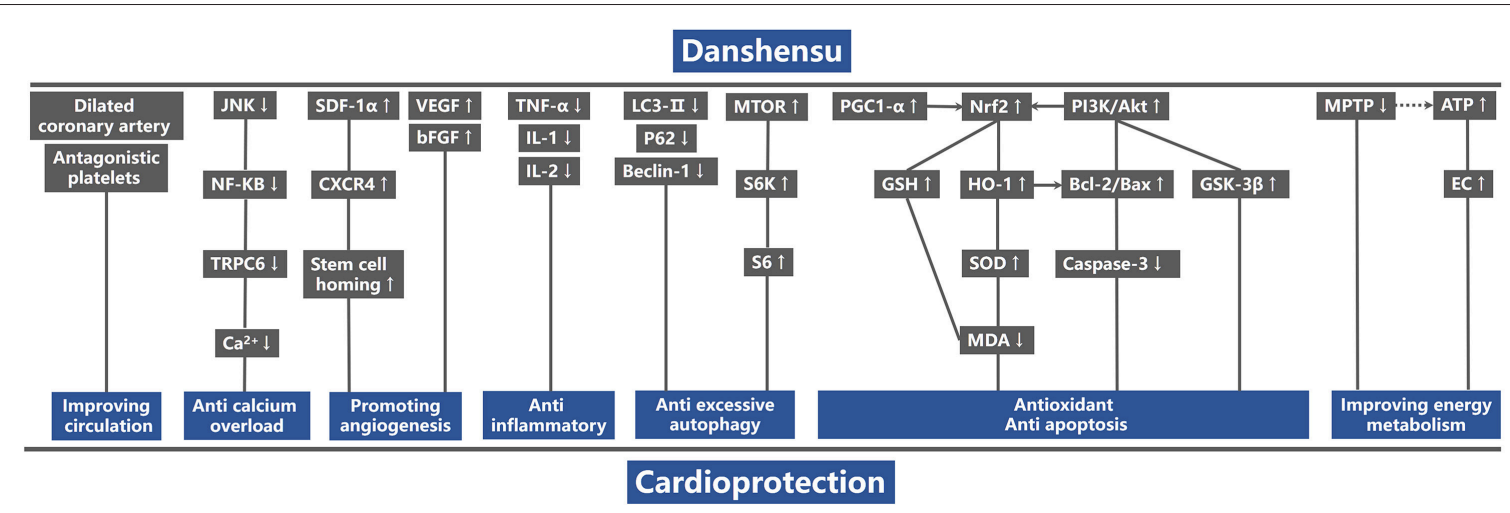

FIGURE 8 | A schematic representation of cardioprotective mechanism of DSS for myocardial ischemic injury. P-Akt, phosphothreonine kinase; P-PI3K, phosphatidylinositol 3-kinase; P-GSK-3 $\beta$, phosphorylated gGlycogen synthase kinase-3 beta; PGC1- $\alpha$, peroxisome proliferator activated receptor $\gamma$ coactivator-1 $\alpha$; $\mathrm{Nif2}$, nuclear factor erythroid 2-related factor 2; HO-1, heme oxygenase-1; GSH, glutathione synthetase; MDA, malondialdehyde; SOD, superoxide dismutase; Bcl-2, B-cell lymphoma-2; Bax, BCL2-associated X protein; TNF- $\alpha$, tumor necrosis factor- $\alpha$; IL-1, Interleukin-1; IL-6, Interleukin-6; SDF-1 $\alpha$, stromal cell-derived factor-1 $\alpha$; CXCR4, C-X-C chemokine receptor type 4; VEGF, vascular endothelial growth factor; bFGF, basic fibroblast growth factor; P-mTOR, phosphorylated mammalian target of rapamycin; P-S6k1, phosphorylated ribosomal protein S6 kinase beta-1; P-S6, phosphorylated ribosomal protein s6; p62, sequestosome-1; LC3, microtubule-associated protein light chain 3; P-JNK, phosphorylated c-Jun N-terminal kinase; NF-KB, nuclear factor-кB; TRPC6, transient receptor potential cation channel, subfamily C, member 6; ATP, adenosine triphosphate; EC, energy charge; MPTP, Mitochondrial permeability transition pore. Solid lines indicate established effects, whereas dashed lines represent putative mechanisms.

\section{Apoptosis rate}

Meta-analysis of 11 studies (Guo et al., 2006; Zhao et al., 2012; Cui G. et al., 2013; Yin et al., 2013; Hu et al., 2016; Meng et al., 2016; Wang et al., 2016, 2017; Gao et al., 2017; Zhang X. et al., 2017; Tang et al., 2018) showed DSS has significant effects on reducing apoptosis rate compared with controls ( $n=74$, SMD: $-3.72,95 \%$ CI: -4.93 to $-2.51, P<0.00001$; heterogeneity $\chi^{2}=17.38, P=$ $0.07, I^{2}=42 \%$; Figure 6B).

\section{Cardioprotective Mechanisms}

Comparing DSS with controls, meta-analysis of 5 studies (Cui G. et al., 2013; Yin et al., 2013; Hu et al., 2016; Wang et al., 2016; Tang et al., 2018) showed significant effects on reducing LDH ( $n=42$, SMD: -4.08 , 95\% CI: -5.55 to $-2.61, P<0.00001$; heterogeneity $\chi^{2}=6.51, P=0.16, I^{2}=39 \%$; Figure 6C); 1 study (Cui G. et al., 2013) for increasing p-PI3K $(P<0.05)$ and phosphorylated Glycogen synthase kinase- 3 beta (p-GSK-3 $\beta$ ) $(P<0.05)$; three studies (Cui G. et al., 2013; Yin et al., 2013; $\mathrm{Hu}$ et al., 2016) for increasing p-AKT $(n=28$, SMD: 4.46, 95\% CI: 2.68-6.24, $P<0.00001$; heterogeneity $\chi^{2}=2.89, P$ $=0.24, I^{2}=31 \%$; Figure 6D); two studies (Wang et al., 2016; Zhang X. et al., 2017) for increasing peroxisome proliferator activated receptor $\gamma$ coactivator- $1 \alpha$ (PGC1- $\alpha)(n=12$, SMD: 3.24, 95\% CI: $0.42-6.05, P<0.05$; heterogeneity $\chi^{2}=1.82, P$ $=0.18, I^{2}=45 \%$; Figure 7A); three studies ( $\mathrm{Hu}$ et al., 2016; Wang et al., 2016; Zhang X. et al., 2017) for increasing Nrf2 ( $n$ $=18$, SMD: $2.47,95 \% \mathrm{CI}: 0.66-4.28, P=0.007$; heterogeneity $\chi^{2}=1.98, P=0.37, I^{2}=0 \%$; Figure $\left.7 \mathbf{B}\right)$; two studies $(\mathrm{Hu}$ et al., 2016; Zhang X. et al., 2017) for increasing HO-1 ( $n=$ 18, SMD: 4.67, 95\% CI: 1.26-8.08, $P<0.00001$; heterogeneity $\chi^{2}=0.13, P=0.72, I^{2}=0 \%$; Figure 7C); one study (Dong et al., 2009) for reducing MDA $(P<0.05)$; 1 study (Fan et al., 2016) for increasing Bcl-2 $(P<0.05)$ and reducing Bax $(P<$
0.05); four studies (Yin et al., 2013; Hu et al., 2016; Wang et al., 2017; Zhang X. et al., 2017) for increasing Bcl-2/Bax $(n=34$, SMD: 5.43, 95\% CI: 3.49-7.38, $P<0.00001$; heterogeneity $\chi^{2}$ $=1.94, P=0.59, I^{2}=0 \%$; Figure 7D); eight studies (Dong et al., 2009; Yin et al., 2013; Fan et al., 2016; Hu et al., 2016; Wang et al., 2016, 2017; Zhang X. et al., 2017; Tang et al., 2018) for reducing caspase-3 $(n=54$, SMD: -4.73 , 95\% CI: -6.39 to $-3.07, P<0.00001$; heterogeneity $\chi^{2}=9.12, P$ $=0.24, I^{2}=23 \%$; Figure 7E); one study (Fan et al., 2016) for increasing p-mTOR $(P<0.01)$; 1 study (Meng et al., 2016) for reducing phosphorylated $\mathrm{c}$-Jun $\mathrm{N}$-terminal kinase (P-JNK) $(P<0.01)$, nuclear factor-кB (NF-KB) $(P<0.01)$ and transient receptor potential cation channel, subfamily $\mathrm{C}$, member 6 (TRPC6) $(P<0.01) .2$ studies (Zhu et al., 1999; Meng et al., 2016) for reducing Calcium $(P<0.01)$; We summarized a schematic representation of cardioprotective mechanism of DSS for myocardial ischemic injury (Figure 8).

\section{DISCUSSION}

\section{Summary of Evidence}

This is the first preclinical systematic review to estimate the efficacy and mechanisms of DSS for Myocardial ischemic injury through both in vivo and in vitro. Thirty-two studies with 473 animals and 134 cells were selected. The quality of the included studies was generally moderate. The present study indicated that DSS exerted cardioprotective function in myocardial ischemic injury, mainly through improving circulation, antioxidant, antiapoptosis, anti-inflammatory, promoting angiogenesis, antiexcessive autophagy, anti-calcium overload and improving energy metabolism. 


\section{Limitations}

First, only English and Chinese database were searched, which may lead to selective bias (Guyatt et al., 2011); Second, negative studies were defficult to publish, which may overstimate the true effecacy of DSS (Franco et al., 2014); Third, no study had used an animal with comobidity, such as diabetes, hypertension, or hyperlipidemia (Heusch, 2017); Finally, primary adult cardiomyocytes were considered to be the suitable subjects to validate cardioprotection in vitro (Lecour et al., 2014), but only one study (Zhu et al., 1999) used it.

\section{Implications}

The damage inflicted on the myocardium during AMI is a complex process, which involves multiple factors. The main mechanisms of injury are energy metabolism disorders, excessive oxy radical, calcium overload, inflammation and mitochondrial dysfunction (Turer and Hill, 2010). Thus, cardiovascular protection drugs generally work through one or combined aspects of the above targets (Heusch, 2015; Ibanez et al., 2015; Qi and Young, 2015). The present study reveals that DSS can reduce MI size, increase cell viability, and reduce apoptosis in myocardial ischemic injury through multiple targets. Hence, our findings provide a preclinical evidence-based approach to develop DSS for acute MI. The possible mechanisms of DSS for myocardial ischemic injury are summarized as follows: (1) improving circulation by expanding the coronary artery and antiplatelet action; (2) antioxidant by promoting the Nrf2 accumulation in nucleus through activation of PI3K/AKTsignaling pathway; (3) anti-apoptosis via upregulation of $\mathrm{Bcl} 2 / \mathrm{Bax}$ and reducing the expression of caspase 3 ; (4) anti-inflammatory; (5) promoting angiogenesis by motivating the homing of stem cells and increasing the expression of VEGF; (6) anti-excessive autophagy via activation of m-TOR signaling pathway; (7) anti-calcium overload by reducing the expression of TRPC6 via inhibiting JNK/NF-KB signaling pathway; (8) improving energy metabolism.

Preclinical systematic review is a novel means that provides robust and informative evidence for animal researches. It contributed to decisions on the utility of further preclinical experiments. Additionally, important information about the translational probability of bench to bed was presented according to the preclinical evidence rating (Sena et al., 2014). In this study, the average CAMARADES score of the included studies is generally medium and still needs further improvement. For animal studies, the main defects are lacking of sample size estimation, blinded induction of model, and blinded assessment of outcome (Zhang K. J. et al., 2017). In addition, all the animal experiments are performed in young and healthy small animals which lack the comorbidities, such as aged, diabetes, hypertension and hyperlipidemia (Van Hout et al., 2016; Heusch, 2017). Cardioprotective effects from a longer-term perspective, such as chronic inflammation, remodeling, heart failure or mortality are not considered (Heusch, 2017). These are main failure factors to translate cardioprotective strategies from the animal study to the clinical practice (Lecour et al., 2014; Heusch, 2017). Reporting guidelines set a detailed checklist of predetermined criteria to make reports of biomedical research more complete and transparent, and thus increasing their value in Scientific exploration, clinical practice and inform policy (Moher et al., 2015). In 2010, the Animal Research: Reporting of in vivo Experiments (ARRIVE) guidelines were published on the initiative of the UK National Centre for the Replacement, Refinement and Reduction of Animals in Research (Kilkenny et al., 2010). The ARRIVE guidelines are organized into twenty sections, covering the key points on reporting study design, experimental procedures, and experimental animals to improve comprehensive and transparent on reporting (Karp et al., 2015). Items 6 and 10 of ARRIVE guideline emphasizes the importance of sample size calculation and blinding. Therefore, we suggest the further animal studies should refer to the ARRIVE and sample size estimation, blinded induction of model, and blinded assessment of outcome should be focused on. In addition, the following factors need to be considered: (1) using large mammals that are closer to humans in anatomy and physiology; (2) experimental animals have comorbidities with aged, diabetes, hypertension, hyperlipidemia or other risk factors; (3) use of primary outcome measures that are closer to clinical practice.

For cells, there are lacking of randomization and blinding (Zhang K. J. et al., 2017). In addition, primary adult cardiomyocytes were considered to be the suitable subjects to validate cardioprotection in vitro (Lecour et al., 2014), however, only one study (Zhu et al., 1999) used it. Thirdly, most cell experiments mimicked the acute I/R injury model using compounds, such as t-BHP, which cannot reflect the complex conditions in vivo (Lecour et al., 2014). Thus, we suggest the further studies focused on randomization and blinding in cell experiments, and it is best to use primary adult cardiomyocytes in ischemic buffer with oxygen-deficient environment, then mimics perfusion using normal buffer with normoxic environment.

Cochrane ROB tool that designed for evaluating the quality of clinical trials was used worldwide (O'Connor et al., 2011). Similarly, some tools have been developed to evaluate the quality of preclinical animal experiments in vivo (Hooijmans et al., 2014; Zhang K. J. et al., 2017). However, there are no quality assessment tools for cell experiments. Thus, we developed an evaluation tool for quality of cell experiments. Detailed description of the assessment tool is as follows: (1) peer review is an inseparable part of science. Non-peer reviewed articles are considered to be of inferior quality and negligible value (Costello et al., 2013). Therefore, it is necessary to use peer review as a part of quality assessment (Table 5A: peer reviewed publication); (2) The use of cell models to simulate clinical disease is important in modern research. However, they do not accurately represent organisms and cannot reproduce the complex interactions in vivo. Thus, it is necessary to select cells that can maximally simulate clinical disease. For example, primary adult cardiomyocytes are most closely related to the characteristics of the adult heart in cell experiments (Lecour et al., 2014). Hence, in the present study, they are the appropriate cells for simulating myocardial ischemic injury. Here we call on researchers to choose the appropriate cells based on actual situation (Table 5B: use appropriate cells to study); (3) quality control of cell lines is essential to ensure the reproducibility of biomedical research. Although cell line certification has been widely recommended for many years, 
misidentification and cross-contamination are still a serious problem. Science and nature, the two top journals, also have repeatedly called attention to this issue (Freedman et al., 2015; Neimark, 2015; Yu M. et al., 2015). Thus, we recommend using cell lines with reliable source or validated by appropriate methods (Table 5C: cell lines with reliable source or validated by appropriate methods); (4) efforts to improve the safety of drugs have always been the key to pharmacological research (Kalgutkar and Dalvie, 2015; Nussinov and Tsai, 2015; Insel et al., 2018). Therefore, special attention should be paid to the toxicity of treatment in preclinical studies (Table 5D: assess toxicity of treatment on cells). (5) The culture environment is crucial for cell growth and subsequent experiments. It is necessary to select a suitable culture environment according to the cell species [Table 5E: culture environment (culture media/sera, $\mathrm{pH} / \mathrm{CO}_{2}$ and temperature)]; (6) the items of random allocation and blinding are essential to RoB (O'Connor et al., 2011; Zhang K. J. et al., 2017) (Table 5F: random allocation to treatment or control; 5G: blinded induction of model; $5 \mathrm{H}$ : blinded assessment of outcome); (7) experiments should have enough sample size to allow for proper statistical analysis and to ensure that the results are robust and reliable. But to avoid waste of resources and prevent exposure of research participants to risk associated with the interventions, the calculation of the sample size necessary to achieve sufficient power is needed (Stokes, 2014) (Table 5I: calculation of the sample size necessary to achieve sufficient power); (8) conflicts of interest are one source of bias. They exist when professional judgment or actions regarding a primary interest may be unduly influenced by a secondary interest (Bero, 2017) (Table 5J: statement of potential conflict of interests).

Improving the transparency and quality of reporting is the imperative of modern scientific research. Numerous publications have emphasized the lack of transparency in reporting, but researches in life sciences, still often lack reporting on the design, conduct and analysis of the experiments (Landis et al., 2012). This resulted in the Consolidated Standards of Reporting Trials (CONSORT) Statement, which was first published in 1996 (Begg et al., 1996), and had been proven to improve the transparency of clinical researches in subsequent implementation (Plint et al., 2006). In 2004, CAMARADES 10-item checklist was first developed to assess the quality of animal experiments (Macleod et al., 2004). Subsequently in 2012, CAMARADES called for transparent reporting to optimize the predictive value of preclinical research (Landis et al., 2012). They recommend that, at minimum, a rigorous study design consist of randomization, blinding, sample-size estimation and the handling of all data should be reported. In the present study, we emphasized the importance of the above recommendations, the items F, $\mathrm{G}, \mathrm{H}$, and I in the cell study checklist are consistent with the CAMARADES claim. In addition, misidentification, crosscontamination, cytotoxicity, and culture environment are core issues widely existed in cell studies. Therefore, we recommend that authors should report on exact source of the cells, the method of verifying the cell type, toxic effect of the drug on the cells, and the detailed data of the cell culture environment. We hope and expect that this tool will fill in the blank areas for preclinical systematic review and meta-analysis of cell studies, subsequently improving the transparency and quality of the in vitro experiments. Furthermore, this tool should be tested for its applicability and validity in practice. We look forward to the feedback from users on this new field of evidence-based cell experiments to make this tool more rigorous, scientific, and valuable.

\section{CONCLUSION}

The findings of present study demonstrated that DSS exerted cardioprotective function in AMI, largely through improving circulation, antioxidant, anti-apoptosis, anti-inflammatory, promoting angiogenesis, anti-excessive autophagy, anticalcium overload and improving energy metabolism. Thus, DSS is a probable candidate for further AMI treatment and clinical trials. In addition, the newly devloped 10-item checklist for assessing methodological quality of cell study that recommened to use the sysmatic review and meta-analysis of cell studies.

\section{AUTHOR CONTRIBUTIONS}

$\mathrm{XB}$ and $\mathrm{QZ}$ contributed equally to this work. XB, QZ, QT, PZ, ZZ, GZ, and YW designed the study. QT, PZ, and ZZ collected the data. XB, QZ, and QT performed all analyses. XB and QZ wrote the manuscript.

\section{FUNDING}

This work was supported by the grant of National Natural Science Foundation of China (81573750/81473491/81173395/H2902).

\section{REFERENCES}

Begg, C., Cho, M., Eastwood, S., Horton, R., Moher, D., Olkin, I., et al. (1996). Improving the quality of reporting of randomized controlled trials. The CONSORT statement. JAMA 276, 637-639. doi: 10.1001/jama.1996.035400800 59030

Bero, L. (2017). Addressing bias and conflict of interest among biomedical researchers. JAMA 317, 1723-1724. doi: 10.1001/jama.2017.3854

Bulluck, H., Yellon, D. M., and Hausenloy, D. J. (2016). Reducing myocardial infarct size: challenges and future opportunities. Heart 102, 341-348. doi: 10.1136/heartjnl-2015-307855
Chen, D. C., Zhang, Y. D., and Pan, Y. F. (2014). Study on danshensu nano-compact liposomes against myocardial ischemia and ischemia-reperfusion injury. Chin. J. Modern. Med. 24, 21-25. doi: 10.3969/j.issn.1005-8982.2014.09.005

Chen, L., Cao, H., and Xiao, J. (2018a). Polyphenols: Absorption, bioavailability, and metabolomics. Polyphenols Properties Recov. Appl. 2, 45-67. doi: 10.1016/C2016-0-05057-X

Chen, L., Teng, H., Fang, T., and Xiao, J. (2016). Agrimonolide from Agrimonia pilosa suppresses inflammatory responses through down-regulation of COX-2/iNOS and inactivation of NF- $\mathrm{BB}$ in lipopolysaccharide-stimulated macrophages. Phytomedicine 23, 846-855. doi: 10.1016/j.phymed.2016.03.016 
Chen, L., Teng, H., Xie, Z., Cao, H., Cheang, W. S., Skalicka-Woniak, K., et al. (2018b). Modifications of dietary flavonoids towards improved bioactivity: An update on structure-activity relationship. Crit. Rev. Food. Sci. Nutr. 58, 513-527. doi: 10.1080/10408398.2016.1196334

Cheng, L. X., Yue, Y. J., Wang, S. X., Nan, Y. F., Zheng, X. H., Qi, M., et al. (2010). Effect of danshensu isopropyl ester on myocardium ischemia/reperfusion injury in isolated rat heart. Chin. Pharmacol. Bull. 26, 1045-1049.

Costello, M. J., Michener, W. K., Gahegan, M., Zhang, Z. Q., and Bourne, P. E. (2013). Biodiversity data should be published, cited, and peer reviewed. Trends. Ecol. Evol. 28, 454-461. doi: 10.1016/j.tree.2013.05.002

Cui, G., Shan, L., Hung, M., Lei, S., Choi, I., Zhang, Z., et al. (2013). A novel danshensu derivative confers cardioprotection via PI3K/Akt and Nrf2 pathways. Int. J. Cardiol. 168, 1349-1359. doi: 10.1016/j.ijcard.2012.12.012

Cui, Q., Chen, Y., Zhang, M., Shan, L., Sun, Y., Yu, P., et al. (2014). Design, synthesis, and preliminary cardioprotective effect evaluation of danshensu derivatives. Chem. Biol. Drug Des. 84, 282-291. doi: 10.1111/cbdd.12312

Cui, Q. B., Shan, L. C., Zhu, H., Yu, P., and Wang, Y. Q. (2013). Modification of alcohol hydroxyl in danshensu structure and evaluation of its primary activity. J. Int. Pharm. Res. 40, 795-800. doi: 10.13220/j.cnki.jipr.2013.06.015

Dong, C., Wang, Y., and Zhu, Y. Z. (2009). Asymmetric synthesis and biological evaluation of danshensu derivatives as anti-myocardial ischemia drug candidates. Bioorg. Med. Chem. 17, 3499-34507. doi: 10.1016/j.bmc.2009.02.065

Fan, G., Yu, J., Asare, P. F., Wang, L., Zhang, H., Zhang, B., et al. (2016). Danshensu alleviates cardiac ischaemia/reperfusion injury by inhibiting autophagy and apoptosis via activation of mTOR signalling. J. Cell. Mol. Med. 20, 1908-1919. doi: $10.1111 /$ jcmm. 12883

Franco, A., Malhotra, N., and Simonovits, G. (2014). Social science. publication bias in the social sciences: unlocking the file drawer. Science 345, 1502-1505. doi: 10.1126/science. 1255484

Freedman, L. P., Gibson, M. C., Ethier, S. P., Soule, H. R., Neve, R. M., and Reid, Y. A. (2015). Reproducibility: changing the policies and culture of cell line authentication. Nat. Methods 12, 493-497. doi: 10.1038/nmeth.3403

Frohlich, G. M., Meier, P., White, S. K., Yellon, D. M., and Hausenloy, D. J. (2013). Myocardial reperfusion injury: looking beyond primary PCI. Eur. Heart. J. 34, 1714-1722. doi: 10.1093/eurheartj/eht090

Gao, Q., Zhao, J., Fan, Z., Bao, J., Sun, D., Li, H., et al. (2017). Cardioprotective effect of danshensu against ischemic/reperfusion injury via c-Subunit of ATP synthase inhibition. Evid. Based. Complement. Alternat. Med. 2017, 7986184. doi: 10.1155/2017/7986184

Guo, Z. Q., Wang, S. R., Zhu, L. Q., Niu, F. L., Huang, Q. F., and Xiao, H. Y. (2006). Effects of danshensu and ligustrazine on angiotensin II induced cardiomyocyte apoptosis in neonatal rats. Chin. J. Integr. Med. Cardio Cerebrovas. Dis. 4, 494-495. doi: 10.3969/j.issn.1672-1349.2006.06.014

Guyatt, G. H., Oxman, A. D., Montori, V., Vist, G., Kunz, R., Brozek, J., et al. (2011). GRADE guidelines: 5 . Rating the quality of evidence-publication bias. J. Clin. Epidemiol. 64, 1277-1282. doi: 10.1016/j.jclinepi.2011.01.011

Hausenloy, D. J., and Yellon, D. M. (2013). Myocardial ischemia-reperfusion injury: a neglected therapeutic target. J. Clin. Invest. 123, 92-100. doi: 10.1172/JCI62874

Heusch, G. (2015). Molecular basis of cardioprotection: signal transduction in ischemic pre-, post-, and remote conditioning. Circ Res. 116, 674-699. doi: 10.1161/CIRCRESAHA.116.305348

Heusch, G. (2017). Critical issues for the translation of cardioprotection. Circ. Res. 120, 1477-1486. doi: 10.1161/CIRCRESAHA.117.310820

Hooijmans, C. R., Rovers, M. M., De Vries, R. B., Leenaars, M., Ritskes-Hoitinga, M., and Langendam, M. W. (2014). SYRCLE's risk of bias tool for animal studies. BMC. Med. Res. Methodol. 14:43. doi: 10.1186/1471-2288-14-43

Hu, T., Wei, G., Xi, M., Yan, J., Wu, X., Wang, Y., et al. (2016). Synergistic cardioprotective effects of danshensu and hydroxysafflor yellow A against myocardial ischemia-reperfusion injury are mediated through the Akt/Nrf2/HO-1 pathway. Int. J. Mol. Med. 38, 83-94. doi: $10.3892 /$ ijmm.2016.2584

Ibanez, B., Heusch, G., Ovize, M., and Vande Werf, F. (2015). Evolving therapies for myocardial ischemia/reperfusion injury. J. Am. Coll. Cardiol. 65, 1454-1471. doi: 10.1016/j.jacc.2015.02.032

Insel, P. A., Amara, S. G., Blaschke, T. F., and Meyer, U. A. (2018). Introduction to the theme "new approaches for studying drug and toxicant action: applications to drug discovery and development". Annu. Rev. Pharmacol. Toxicol. 58, 33-36. doi: 10.1146/annurev-pharmtox-092617-121952

Jiang, W. D., Chen, Y. H., Wang, Y. P., Dong, Z. T., Yuan, L. X., Lu, Y. Q., et al. (1982). Effects of danshensu and two other water-soluble salvia miltiorrhiza compounds on myocardial ischemia and coronary artery. Fudan Univ. J. Med. Sci. 9, 13-20.

Kalgutkar, A. S., and Dalvie, D. (2015). Predicting toxicities of reactive metabolite-positive drug candidates. Annu. Rev. Pharmacol. Toxicol. 55, 35-54. doi: 10.1146/annurev-pharmtox-010814-124720

Karp, N. A., Meehan, T. F., Morgan, H., Mason, J. C., Blake, A., Kurbatova, N., et al. (2015). Applying the ARRIVE guidelines to an in vivo database. PLoS Biol. 13:e1002151. doi: 10.1371/journal.pbio.1002151

Kilkenny, C., Browne, W. J., Cuthill, I. C., Emerson, M., and Altman, D. G. (2010). Improving bioscience research reporting: the ARRIVE guidelines for reporting animal research. J. Pharmacol. Pharmacother. 1, 94-99. doi: 10.4103/0976-500X.72351

Landis, S. C., Amara, S. G., Asadullah, K., Austin, C. P., Blumenstein, R., Bradley, E. W., et al. (2012). A call for transparent reporting to optimize the predictive value of preclinical research. Nature 490, 187-191. doi: 10.1038/nature 11556

Lecour, S., Bøtker, H. E., Condorelli, G., Davidson, S. M., Garcia-Dorado, D., Engel, F. B., et al. (2014). ESC working group cellular biology of the heart: position paper: improving the preclinical assessment of novel cardioprotective therapies. Cardiovasc. Res. 104, 399-411. doi: 10.1093/cvr/cvu225

Li, H., Song, F., Duan, L. R., Sheng, J. J., Xie, Y. H., Yang, Q., et al. (2016). Paeonol and danshensu combination attenuates apoptosis in myocardial infarcted rats by inhibiting oxidative stress: roles of Nrf2/HO-1 and PI3K/Akt pathway. Sci. Rep. 6:23693. doi: 10.1038/srep23693

Li, H., Xie, Y. H., Yang, Q., Wang, S. W., Zhang, B. L., Wang, J. B., et al. (2012). Cardioprotective effect of paeonol and danshensu combination on isoproterenol-induced myocardial injury in rats. PLOS ONE 7:e48872. doi: 10.1371/journal.pone.0048872

Li, X. Q., Ma, L. Y., and Zhu, S. J. (1996). Changes of high-energy phosphate compounds during myocardial ischemia/reperfusion injury in rats and the protective effect of danshensu. Chin. J. Pathophysiol. 12, 270-273.

Liberati, A., Altman, D. G., Tetzlaff, J., Mulrow, C., Gøtzsche, P. C., Ioannidis, J. P., et al. (2009). The PRISMA statement for reporting systematic reviews and meta-analyses of studies that evaluate healthcare interventions: explanation and elaboration. BMJ 339:b2700. doi: 10.1136/bmj.b2700

Lu, W. Q., Zhang, G. Q., Chen, D. G., and Zhang, C. (2010). Protective effect of danshensu on acute myocardial ischemia in rats. J. Pharmaceut. Pract. 28, 279-281. doi: 10.3969/j.issn.1006-0111.2010.04.013

Macleod, M. R., O'Collins, T., Howells, D. W., and Donnan, G. A. (2004). Pooling of animal experimental data reveals influence of study design and publication bias. Stroke 35, 1203-1208. doi: 10.1161/01.STR.0000125719.25853.20

Meng, Y., Li, W. Z., Shi, Y. W., Zhou, B. F., Ma, R., and Li, W. P. (2016). Danshensu protects against ischemia/reperfusion injury and inhibits the apoptosis of H9c2 cells by reducing the calcium overload through the p-JNK-NF- $\mathrm{KB}-\mathrm{TRPC6}$ pathway. Int. J. Mol. Med. 37, 258-266. doi: 10.3892/ijmm.2015.2419

Moher, D., Avey, M., Antes, G., and Altman, D. G. (2015). The National Institutes of Health and guidance for reporting preclinical research. BMC Med. 13:34. doi: 10.1186/s12916-015-0284-9

Mozaffarian, D., Benjamin, E. J., Go, A. S., Arnett, D. K., Blaha, M. J., Cushman, M., et al. (2016). Heart disease and strokestatistics--2016 update: a report from the american heart association. Circulation 133, e38-e360. doi: 10.1161/CIR.0000000000000350

Neimark, J. (2015). Line of attack. Science 347, 938-940. doi: $10.1126 /$ science 347.6225 .938

Nussinov, R., and Tsai, C. J. (2015). The design of covalent allosteric drugs. Annu. Rev. Pharmacol. Toxicol. 55, 249-267. doi: 10.1146/annurev-pharmtox-010814-124401

O'Connor, D., Green, S., and Higgins, J. P. T. (2011). "Chapter 5: defining the reviewquestion and developing criteria for including studies," in Cochrane Handbook for Systematic Reviews of Interventions Version 510, eds J. P. T. Higgins, and S. Green (London: The Cochrane Collaboration), 97-107.

Plint, A. C., Moher, D., Morrison, A., Schulz, K., Altman, D. G., Hill, C., et al. (2006). Does the CONSORT checklist improve the quality of reports of randomised controlled trials? A systematic review. Med. J. Aust. 185, 263-267. 
Qi, D., and Young, L. H. (2015). AMPK: energy sensor and survival mechanism in the ischemic heart. Trends. Endocrinol. Metab. 26, 422-429. doi: 10.1016/j.tem.2015.05.010

Quan, W., Zhou, D., Guo, C., Wang, Y. H., Zhu, Y. R., Guan, Y., et al. (2012). Protective effect of danshensu sodium on myocardial ischemia/reperfusion injury by inhibiting the inflammatory response. Cent. South. Pharm. 10, 885-888. doi: 10.3969/j.issn.1672.2981.2012.12.002

Sena, E. S., Currie, G. L., McCann, S. K., Macleod, M. R., and Howells, D. W. (2014). Systematic reviews and meta-analysis of preclinical studies: why perform them and how to appraise them critically. J. Cereb. Blood Flow. Metab. 34, 737-742. doi: $10.1038 / \mathrm{jcbfm} .2014 .28$

Song, Q., Chu, X., Zhang, X., Bao, Y., Zhang, Y., Guo, H., et al. (2016). Mechanisms underlying thecardioprotective effect of salvianic acid A against isoproterenol-induced myocardial ischemia injury in rats: possible involvement of L-type calcium channels and myocardial contractility. J. Ethnopharmacol. 189, 157-164. doi: 10.1016/j.jep.2016.05.038

Stokes, L. (2014). Sample size calculation for a hypothesis test. JAMA 312, 180-181. doi: 10.1001/jama.2014.8295

Tang, F., Zhou, X., Wang, L., Shan, L., Li, C., Zhou, H., et al. (2018). A novel compound DT-010 protects against doxorubicin-induced cardiotoxicity in zebrafish and $\mathrm{H} 9 \mathrm{c} 2$ cells by inhibiting reactive oxygen species-mediated apoptotic and autophagic pathways. Eur. J. Pharmacol. 820, 86-96. doi: 10.1016/j.ejphar.2017.12.021

Tang, L. H., Wang, X. M., and Liang, D. Q. (1989). Protective effect of Danshensu on myocardial ischemia/reperfusion injury in rats. Chin. J. Pathophysiol. 5, 65-69.

Teng, H., and Chen, L. (2018). Polyphenols and bioavailability: an update. Crit. Rev. Food. Sci. Nutr. 6:0. doi: 10.1080/10408398.2018.1437023

Teng, H., Chen, L., and Song, H. (2016a). The potential beneficial effects of phenolic compounds isolated from A. Ledeb on 2 hepatic HepG2 insulinresistant cells. Food. Funct. 7, 4400-4409. doi: 10.1039/C5FO01067E

Teng, H., Huang, Q., and Chen, L. (2016b). Inhibition of cell proliferation and triggering of apoptosis by agrimonolide through MAP kinase (ERK and p38) pathways in human gastric cancer AGS cells. Food. Funct. 7, 4605-4613. doi: 10.1039/C6FO00715E

Turer, A. T., and Hill, J. A. (2010). Pathogenesis of myocardial ischemiareperfusion injury and rationale for therapy. Am. J. Cardiol. 106, 360-368. doi: 10.1016/j.amjcard.2010.03.032

Van Hout, G. P., Jansen of Lorkeers, S. J., Wever, K. E., Sena, E. S., Kouwenberg, L. H., van Solinge, W. W., et al. (2016). Translational failure of anti-inflammatory compounds for myocardial infarction: a meta-analysis of large animal models. Cardiovasc. Res. 109, 240-248. doi: 10.1093/cvr/cvv239

Wang, L., Zhang, X., Chan, J. Y., Shan, L., Cui, G., Cui, Q., et al. (2016). A novel danshensu derivative prevents cardiac dysfunction and improves the chemotherapeutic efficacy of doxorubicin in breast cancer cells. J. Cell. Biochem. 117, 94-105. doi: 10.1002/jcb.25253

Wang, Y., Zhang, X., Xu, C., Zhang, G., Zhang, Z., Yu, P., et al. (2017). Synthesis and biological evaluation of danshensu and tetramethylpyrazine conjugates as cardioprotective agents. Chem. Pharm. Bull. (Tokyo) 65, 381-388. doi: 10.1248/cpb.c16-00839

Wei, G., Duan, J. L., Yin, Y., Wang, Y. H., and Wen, A. D. (2016). Angiogenesis and anti-myocardial infarction effect of danshensu and hydroxysafflor yellow A in rats. LiShiZhen Med. Mater. Med. Res. 27, 2576-2578. doi: 10.3969/j.issn.1008-0805.2016.11.007

Xiang, Z., Jing, L. L., Liu, W. C., Wang, P. A., Gao, F., and Sun, X. L. (2009). Synthesis of isosorbide mononitrate mononitrate and its protective effect on myocardial ischemia/reperfusion in rats. Negative 30, 1257-1261. doi: 10.3321/j.issn.1000-2790.2009.14.003

Xu, B. H., Cui, Q. B., Zhu, H., Zhao, Z. C., and Shan, L. C. (2013). Study on synthesis and primaryactivity of new danshensu derivatives. Cent. South. Pharm. 11, 338-341. doi: 10.7539/j.issn.1672-2981.2013.05.005

Yin, Y., Duan, J., Guo, C., Wei, G., Wang, Y., Guan, Y., et al. (2017). Danshensu accelerates angiogenesis after myocardial infarction in rats and promotes the functions of endothelial progenitor cells through SDF1 $\alpha /$ CXCR4 axis. Eur. J. Pharmacol. 814, 274-282. doi: 10.1016/j.ejphar.2017. 08.035

Yin, Y., Guan, Y., Duan, J., Wei, G., Zhu, Y., Quan, W., et al. (2013). Cardioprotective effect of danshensu against myocardial ischemia/reperfusion injury and inhibits apoptosis of H9c2 cardiomyocytes via Akt and ERK1/2 phosphorylation. Eur. J. Pharmacol. 699, 219-226. doi: 10.1016/j.ejphar.2012.11.005

Yu, J., Wang, L., Akinyi, M., Li, Y., Duan, Z., Zhu, Y., et al. (2015). Danshensu protects isolated heart against ischemia reperfusion injury through activation of Akt/ERK1/2/Nrf2 signaling. Int. J. Clin. Exp. Med. 8, 14793-14804.

Yu, M., Selvaraj, S. K., Liang-Chu, M. M., Aghajani, S., Busse, M., Yuan, J., et al. (2015). A resource for cell line authentication, annotation and quality control. Nature. 520, 307-311. doi: 10.1038/nature14397

Zhang, K. J., Zhu, J. Z., Bao, X. Y., Zheng, Q., Zheng, G. Q., and Wang, Y. (2017). Shexiang baoxin pills for coronary heart disease in animal models: preclinical evidence and promoting angiogenesis mechanism. Front Pharmacol. 8:404. doi: 10.3389/fphar.2017.00404

Zhang, L., Chang, B. B., Cao, W. W., Cao, Y., Yang, W. L., and Liu, X. Q. (2010). Effects of danshensu on myocardial energy metabolism in isolated heart ischemic reperfusion rats. J. Chin. Pharmaceut. Univ. 41, 278-282. doi: 10.11665/j.issn.1000-5048.20100318

Zhang, N., Dong, M., Luo, Y., Zhao, F., and Li, Y. (2018). Danshensu prevents hypoxic pulmonary hypertension in rats by inhibiting the proliferation of pulmonary artery smooth muscle cells via TGF- $\beta$-smad3associated pathway. Eur. J. Pharmacol. 820, 1-7. doi: 10.1016/j.ejphar.2017. 12.010

Zhang, X., Hu, H., Luo, J., Deng, H., Yu, P., Zhang, Z., et al. (2017). A novel danshensu-tetramethylpyrazine conjugate DT-010 provides cardioprotection through the PGC-1 $\alpha / \mathrm{Nrf} / \mathrm{HO}-1$ pathway. Biol. Pharm. Bull. 40, 1490-1498. doi: 10.1248/bpb.b17-00313

Zhao, H. S., Zhang, M. J., Xu, B. H., Guo, L., Fu, H. L., and Shan, L. C. (2012). Study on anti-cardiomyocyte apoptosis effect of new danshensu derivatives. Cent. South. Pharm. 10, 418-421. doi: 10.3969/j.issn.1672-2981.2012.06.006

Zhu, P. X., Liu, G. M., Yang, Z. H., Wang, X. M., and Ma, L. Y. (1999). Effects of danshensu and SOD on changes of free calcium concentration in oxygen abnormal myocardial cells. Chin. J. Pathophysiol. 15, 877-879.

Conflict of Interest Statement: The authors declare that the research was conducted in the absence of any commercial or financial relationships that could be construed as a potential conflict of interest.

Copyright (c) 2018 Bao, Zheng, Tong, Zhu, Zhuang, Zheng and Wang. This is an open-access article distributed under the terms of the Creative Commons Attribution License (CC BY). The use, distribution or reproduction in other forums is permitted, provided the original author(s) and the copyright owner(s) are credited and that the original publication in this journal is cited, in accordance with accepted academic practice. No use, distribution or reproduction is permitted which does not comply with these terms. 